\title{
One multidimensional version of the Darboux first problem for one class of semilinear second order hyperbolic systems
}

\author{
S. Kharibegashvili and B. Midodashvili
}

\begin{abstract}
One multidimensional version of the Darboux first problem for one class of semilinear second order hyperbolic systems is investigated. The questions on local and global solvability and nonexistence of a global solution of this problem are considered.
\end{abstract}

Mathematics Subject Classification. 35L51, 35L71.

Keywords. Multidimensional version of the Darboux first problem, Semilinear second order hyperbolic systems, Local and global solvability, Nonexistence of a global solution.

\section{Introduction}

In the Euclidean space $\mathbb{R}^{n+1}$ of independent variables $x=\left(x_{1}, x_{2}, \ldots, x_{n}\right)$ and $t$ consider a second order semilinear hyperbolic system of the form

$$
\square u_{i}+f_{i}\left(u_{1}, \ldots, u_{N}\right)=F_{i}, \quad i=1, \ldots, N,
$$

where $f=\left(f_{1}, \ldots, f_{N}\right), F=\left(F_{1}, \ldots, F_{N}\right)$ are given, and $u=\left(u_{1}, \ldots, u_{N}\right)$ is an unknown real vector-functions, $n \geq 2, N \geq 2, \square:=\frac{\partial^{2}}{\partial t^{2}}-\Delta, \Delta:=\sum_{i=1}^{n} \frac{\partial^{2}}{\partial x_{i}^{2}}$.

Denote by $D: t>|x|, x_{n}>0$ the half of a light cone of the future bounded by the part $S^{0}: \partial D \cap\left\{x_{n}=0\right\}$ of hyperplane $x_{n}=0$ and the half $S: t=|x|, x_{n} \geq 0$ of the characteristic conoid $C: t=|x|$ of the system (1.1). Let $D_{T}:=\{(x, t) \in D: t<T\}, S_{T}^{0}:=\left\{(x, t) \in S^{0}: t \leq T\right\}$, $S_{T}:=\{(x, t) \in S: t \leq T\}, T>0$.

For the system of equations (1.1) consider a problem on finding a solution $u(x, t)$ of this system by the following boundary conditions

$$
\left.\frac{\partial u}{\partial x_{n}}\right|_{S_{T}^{0}}=0,\left.u\right|_{S_{T}}=g
$$


where $g=\left(g_{1}, \ldots, g_{N}\right)$ is a given vector-function on $S_{T}$. In the case when $T=\infty$ we have $D_{\infty}=D, S_{\infty}^{0}=S^{0}$ and $S_{\infty}=S$.

The problem (1.1), (1.2) represents a multidimensional version of the Darboux first problem for the system (1.1), when one part of the problem data support represents a characteristic manifold, while another part is of time type manifold [1, p. 228, 233].

Questions on the existence and nonexistence of global solution of the Cauchy problem for semilinear scalar equations of the form (1.1) with initial conditions $\left.u\right|_{t=0}=u_{0},\left.\frac{\partial u}{\partial t}\right|_{t=0}=u_{1}$ have been considered by many authors (see, e.g. [2-11]). As it is known for second order scalar linear hyperbolic equations multidimensional versions of the Darboux first problem are well-posed and they are globally solvable in suitable function spaces [12-17]. In regard to the multidimensional problem (1.1), (1.2) for scalar case, i.e. when $N=1$, in the case of nonlinearity of the form $f(u)=\lambda|u|^{p} u$, as it is shown in the paper [18], depending on the sign of the parameter $\lambda$ and the values of power exponent $p$, in some cases the problem (1.1), (1.2) is globally solvable, while in other cases it is not globally solvable. Another multidimensional version of the Darboux first problem for scalar semilinear equation of the form (1.1), where instead of the boundary condition $\left.\frac{\partial u}{\partial x_{n}}\right|_{S_{T}^{0}}=0$ in (1.2) is taken $\left.u\right|_{S_{T}^{0}}=0$, is considered in the work [19]. Worthy to note also that the multidimensional version for the Darboux second problem for scalar semilinear equation of the form (1.1) is studied in the work [20].

In the present work we give certain conditions for the nonlinear vectorfunction $f=f(u)$ from (1.1), which fulfilment ensures local or global solvability of the problem (1.1), (1.2), while in some cases it will not have global solution, though it will be locally solvable.

The paper is organized in the following way. In the Sect. 2 we define a strong generalized solution of the problem (1.1), (1.2) of the class $W_{2}^{1}$ in the domain $D_{T}$ and a global solution of this problem of the same class in the domain $D_{\infty}$. In the Sect. 3, with some restrictions on the power of nonlinearity of the vector function $f$, we consider the cases of local and global solvability of the problem (1.1), (1.2) of the class $W_{2}^{1}$. In Sect. 4 we give conditions for the vector-function $f$, which provide the existence and uniqueness of a global solution of the class $W_{2}^{1}$ to this problem. Finally, in the Sect. 5, at some additional conditions on the vector-functions $f, F$ and $g$, we prove the nonexistence of a global solution of the problem (1.1), (1.2) of the class $W_{2}^{1}$ in the domain $D_{\infty}$.

\section{Definition of a generalized solution of the problem (1.1), (1.2) in $D_{T}$ and $D_{\infty}$}

Let $\stackrel{0}{C}^{2}\left(\bar{D}_{T}, S_{T}^{0}, S_{T}\right):=\left\{u \in C^{2}\left(\bar{D}_{T}\right):\left.\frac{\partial u}{\partial x_{n}}\right|_{S_{T}^{0}}=0,\left.u\right|_{S_{T}}=0\right\}$.

Let also $\stackrel{0}{W} \underset{2}{1}\left(D_{T}, S_{T}\right):=\left\{u \in W_{2}^{1}\left(D_{T}\right):\left.u\right|_{S_{T}}=0\right\}$, where $W_{2}^{k}(\Omega)$ is the Sobolev space consisting of the elements of $L_{2}(\Omega)$ having up to $k$-th order 
generalized derivatives from $L_{2}(\Omega)$, inclusively. Here the equality $\left.u\right|_{S_{T}}=0$ must be understood in the sense of the trace theory [21, p. 71].

Below under belonging of the vector $v=\left(v_{1}, \ldots, v_{N}\right)$ to some space $X$ we mean belonging of each component $v_{i}, 1 \leq i \leq N$, of this vector to the same space $X$. In accordance with this, for simplicity of record, where this will not cause misunderstanding, instead of $v=\left(v_{1}, v_{2}, \ldots, v_{N}\right) \in[X]^{N}$ we use the record $v \in X$.

Rewrite the system of equations (1.1) in the form of one vector equation

$$
L u:=\square u+f(u)=F .
$$

Together with the boundary conditions (1.2) we consider corresponding homogeneous boundary conditions

$$
\left.\frac{\partial u}{\partial x_{n}}\right|_{S_{T}^{0}}=0,\left.u\right|_{S_{T}}=0 .
$$

Below, on the nonlinear vector-function $f=\left(f_{1}, \ldots, f_{N}\right)$ in (1.1) we impose the following requirement

$$
f \in C\left(\mathbb{R}^{N}\right), \quad|f(u)| \leq M_{1}+M_{2}|u|^{\alpha}, \quad \alpha=\text { const } \geq 0, \quad u \in \mathbb{R}^{N},
$$

where $|\cdot|$ is a norm in the space $\mathbb{R}^{N}, M_{i}=$ const $\geq 0, i=1,2$.

Remark 2.1. The embedding operator $I:\left[W_{2}^{1}\left(D_{T}\right)\right]^{N} \rightarrow\left[L_{q}\left(D_{T}\right)\right]^{N}$ represents a linear continuous compact operator for $1<q<\frac{2(n+1)}{n-1}$, when $n>1$ [21, p. 86]. At the same time the Nemitski operator $K:\left[L_{q}\left(D_{T}\right)\right]^{N} \rightarrow\left[L_{2}\left(D_{T}\right)\right]^{N}$, acting by the formula $K u=f(u)$, where $u=\left(u_{1}, \ldots, u_{N}\right) \in\left[L_{q}\left(D_{T}\right)\right]^{N}$, and the vector-function $f=\left(f_{1}, \ldots, f_{N}\right)$ satisfies the condition (2.3).0 is continuous and bounded for $q \geq 2 \alpha$ [22, p. 349], [23, pp. 66, 67]. Thus, if $\alpha<\frac{n+1}{n-1}$, i.e. $2 \alpha<\frac{2(n+1)}{n-1}$, then there exists number $q$ such that $1<q<\frac{2(n+1)}{n-1}$ and $q \geq 2 \alpha$. Therefore, in this case the operator

$$
K_{0}=K I:\left[W_{2}^{1}\left(D_{T}\right)\right]^{N} \rightarrow\left[L_{2}\left(D_{T}\right)\right]^{N}
$$

will be continuous and compact. It is clear that from $u=\left(u_{1}, \ldots, u_{N}\right) \in$ $W_{2}^{1}\left(D_{T}\right)$ it follows that $f(u) \in L_{2}\left(D_{T}\right)$ and, if $u^{m} \rightarrow u$ in the space $W_{2}^{1}\left(D_{T}\right)$, then $f\left(u^{m}\right) \rightarrow f(u)$ in the space $L_{2}\left(D_{T}\right)$.

Definition 2.1. Let $f=\left(f_{1}, \ldots, f_{N}\right)$ satisfy the condition $(2.3)$, where $0 \leq \alpha<$ $\frac{n+1}{n-1}, F=\left(F_{1}, \ldots, F_{N}\right) \in L_{2}\left(D_{T}\right)$ and $g=\left(g_{1}, \ldots, g_{N}\right) \in W_{2}^{1}\left(S_{T}\right)$. We call the vector-function $u=\left(u_{1}, \ldots, u_{N}\right) \in W_{2}^{1}\left(D_{T}\right)$ a strong generalized solution of the problem (1.1), (1.2) of the class $W_{2}^{1}$ in the domain $D_{T}$ if there exists a sequence of vector-functions $u^{m} \in C^{2}\left(\bar{D}_{T}\right)$ such that $\left.\frac{\partial u^{m}}{\partial x_{n}}\right|_{S_{T}^{0}}=0, u^{m} \rightarrow u$ in the space $W_{2}^{1}\left(D_{T}\right), L u^{m} \rightarrow F$ in the space $L_{2}\left(D_{T}\right)$ and $\left.u^{m}\right|_{S_{T}} \rightarrow g$ in the space $W_{2}^{1}\left(S_{T}\right)$. Convergence of the sequence $\left\{f\left(u^{m}\right)\right\}$ to $f(u)$ in the space $L_{2}\left(D_{T}\right)$ when $u^{m} \rightarrow u$ in the space $W_{2}^{1}\left(D_{T}\right)$ follows from the Remark 2.1 When $g=0$, i.e. in the case of homogeneous boundary conditions (2.2), we assume that $u^{m} \in \stackrel{0}{C}^{2}\left(\bar{D}_{T}, S_{T}^{0}, S_{T}\right)$. Then, it is clear that $u \in \stackrel{0}{W} \underset{2}{1}\left(D_{T}, S_{T}\right)$. 
It is obvious, that the classical solution $u \in C^{2}\left(\bar{D}_{T}\right)$ of the problem (1.1), (1.2) is a strong generalized solution of the class $W_{2}^{1}$ in the domain $D_{T}$ in the sense of the Definition 2.1.

Remark 2.2. It is easy to verify that if $u \in C^{2}\left(\bar{D}_{T}\right)$ is a classical solution of the problem (1.1), (1.2), then scalarly multiplying the both parts of the system $(2.1)$ by any test vector-function $\varphi=\left(\varphi_{1}, \ldots, \varphi_{N}\right) \in C^{1}\left(\bar{D}_{T}\right)$, satisfying the condition $\left.\varphi\right|_{t=T}=0$, after integration by parts, we receive the following equality

$$
\begin{gathered}
\int_{D_{T}}\left[-u_{t} \varphi_{t}+\nabla u \nabla \varphi\right] d x d t=-\int_{D_{T}} f(u) \varphi d x d t \\
+\int_{D_{T}} F \varphi d x d t-\int_{S_{T}^{0} \cup S_{T}} \frac{\partial u}{\partial \aleph} \varphi d s,
\end{gathered}
$$

where $\frac{\partial}{\partial \aleph}=\nu_{n+1} \frac{\partial}{\partial t}-\sum_{i=1}^{n} \nu_{i} \frac{\partial}{\partial x_{i}}$ is the derivative with respect to the conormal, $\nu=\left(\nu_{1}, \ldots, \nu_{n}, \nu_{n+1}\right)$ is the unit vector of the outer normal to $\partial D_{T}$, and $\nabla=\left(\partial / \partial x_{1}, \ldots, \partial / \partial x_{n}\right)$. Taking into account that $\left.\frac{\partial}{\partial \aleph}\right|_{S_{T}^{0}}=\frac{\partial}{\partial x_{n}}$ and $S_{T}$ represents a characteristic manifold, on which the operator $\frac{\partial}{\partial \aleph}$ is a inner differential operator, from (1.2) we have $\left.\frac{\partial u}{\partial \aleph}\right|_{S_{T}^{0}}=0,\left.\frac{\partial u}{\partial \aleph}\right|_{S_{T}}=\left.\frac{\partial g}{\partial \aleph}\right|_{S_{T}}$. Therefore, the equality (2.5) takes the form

$$
\begin{aligned}
\int_{D_{T}}\left[-u_{t} \varphi_{t}+\nabla u \nabla \varphi\right] d x d t= & -\int_{D_{T}} f(u) \varphi d x d t \\
& +\int_{D_{T}} F \varphi d x d t-\int_{S_{T}} \frac{\partial g}{\partial \aleph} \varphi d s
\end{aligned}
$$

It is easy to see that the equality (2.6) is valid also for any vector-function $\varphi=\left(\varphi_{1}, \ldots, \varphi_{N}\right) \in W_{2}^{1}\left(D_{T}\right)$ such that $\left.\varphi\right|_{t=T}=0$ in the sense of the trace theory. Note that the equality (2.6) is valid also for a strong generalized solution $u \in W_{2}^{1}\left(D_{T}\right)$ of the problem (1.1), (1.2) of the class $W_{2}^{1}$ in the domain $D_{T}$ in the sense of the Definition 2.1. Indeed, if $u^{m} \in C^{2}\left(D_{T}\right)$ is a sequence of vector-functions from the Definition 2.1, then writing equality (2.6) for $u=u^{m}$ and passing to the limit for $m \rightarrow \infty$, we receive (2.6). Note also that the equality (2.6), valid for any test vector-function $\varphi \in W_{2}^{1}\left(D_{T}\right)$, satisfying the condition $\left.\varphi\right|_{t=T}=0$, can be put in the basis of the definition of a weak generalized solution $u \in W_{2}^{1}\left(D_{T}\right)$ of the problem (1.1), (1.2) of the class $W_{2}^{1}$ in the domain $D_{T}$.

Definition 2.2. Let $f$ satisfy the condition (2.3), where $0 \leq \alpha<\frac{n+1}{n-1} ; F \in$ $L_{2, l o c}\left(D_{\infty}\right), g \in W_{2, l o c}^{1}\left(S_{\infty}\right)$ and $\left.F\right|_{D_{T}} \in L_{2}\left(D_{T}\right),\left.g\right|_{S_{T}} \in W_{2}^{1}\left(S_{T}\right)$ for any $T>0$. We say that the problem (1.1), (1.2) is locally solvable in the class $W_{2}^{1}$ if there exists a number $T_{0}=T_{0}(F, g)>0$ such that for any $T<T_{0}$ this problem has a strong generalized solution of the class $W_{2}^{1}$ in the domain $D_{T}$ in the sense of the Definition 2.1. 
Definition 2.3. Let $f$ satisfy the condition (2.3), where $0 \leq \alpha<\frac{n+1}{n-1} ; F \in$ $L_{2, l o c}\left(D_{\infty}\right), g \in W_{2, l o c}^{1}\left(S_{\infty}\right)$ and $\left.F\right|_{D_{T}} \in L_{2}\left(D_{T}\right),\left.g\right|_{S_{T}} \in W_{2}^{1}\left(S_{T}\right)$ for any $T>0$. We say that the problem (1.1), (1.2) is globally solvable in the class $W_{2}^{1}$ if for any $T>0$ this problem has a strong generalized solution of the class $W_{2}^{1}$ in the domain $D_{T}$ in the sense of the Definition 2.1.

Definition 2.4. Let $f$ satisfy the condition (2.3), where $0 \leq \alpha<\frac{n+1}{n-1} ; F \in$ $L_{2, l o c}\left(D_{\infty}\right), g \in W_{2, l o c}^{1}\left(S_{\infty}\right)$ and $\left.F\right|_{D_{T}} \in L_{2}\left(D_{T}\right),\left.g\right|_{S_{T}} \in W_{2}^{1}\left(S_{T}\right)$ for any $T>0$. Vector-function $u=\left(u_{1}, \ldots, u_{N}\right) \in W_{2, l o c}^{1}\left(D_{\infty}\right)$ is called a global strong generalized solution of the problem (1.1), (1.2) of the class $W_{2}^{1}$ in the domain $D_{\infty}$ if for any $T>0$ the vector-function $\left.u\right|_{D_{T}}$ belongs to the space $W_{2}^{1}\left(D_{T}\right)$ and represents a strong generalized solution of the problem (1.1), (1.2) of the class $W_{2}^{1}$ in the domain $D_{T}$ in the sense of the Definition 2.1.

Remark 2.3. Reasoning used in the proof of the equality (2.6) makes it possible to conclude that the global strong generalized solution $u=\left(u_{1}, \ldots, u_{N}\right)$ of the problem (1.1), (1.2) of the class $W_{2}^{1}$ in the domain $D_{\infty}$ in the sense of the Definition 2.4 satisfies the following integral equality

$$
\begin{aligned}
\int_{D_{\infty}}\left[-u_{t} \varphi_{t}+\nabla u \nabla \varphi\right] d x d t= & -\int_{D_{\infty}} f(u) \varphi d x d t \\
& +\int_{D_{\infty}} F \varphi d x d t-\int_{S_{\infty}} \frac{\partial q}{\partial \aleph} \varphi d s
\end{aligned}
$$

for any test vector-function $\varphi=\left(\varphi_{1}, \ldots, \varphi_{N}\right) \in C^{1}\left(D_{\infty}\right)$, which is finite with respect to the variable $r=\left(t^{2}+|x|^{2}\right)^{1 / 2}$, i.e. $\varphi=0$ for $r>r_{0}=$ const $>0$.

\section{Some cases of local and global solvability of the problem (1.1), (1.2) in the class $W_{2}^{1}$}

For simplicity we consider the case when the boundary conditions (1.2) are homogeneous. In this case the problem (1.1), (1.2) can be written in the form (2.1), (2.2).

Remark 3.1. Before we consider the solvability of the problem (2.1), (2.2) let us consider the same question for a linear case, when vector-function $f=0$ in (2.1), i.e. for the problem

$$
\begin{gathered}
L_{0} u:=\square u=F(x, t), \quad(x, t) \in D_{T}, \\
\left.\frac{\partial u}{\partial x_{n}}\right|_{S_{T}^{0}}=0,\left.\quad u\right|_{S_{T}}=0 .
\end{gathered}
$$

For the problem (3.1), (3.2), by analogy to that in the Definition 2.1 for the problem (1.1), (1.2), we introduce a notion of the strong generalized solution $u=\left(u_{1}, \ldots, u_{N}\right)$ of the class $W_{2}^{1}$ in the domain $D_{T}$ for $F=\left(F_{1}, \ldots, F_{N}\right) \in$ $L_{2}\left(D_{T}\right)$, i.e. for vector-function $u=\left(u_{1}, \ldots, u_{N}\right) \in \stackrel{0}{W}{ }_{2}^{1}\left(D_{T}, S_{T}\right)$, for which 
there exists a sequence of vector-functions $u^{m}=\left(u_{1}^{m}, \ldots, u_{N}^{m}\right) \in \stackrel{0}{C}^{2}\left(\bar{D}_{T}, S_{T}^{0}\right.$, $\left.S_{T}\right)$, such that

$$
\lim _{m \rightarrow \infty}\left\|u^{m}-u\right\|_{W_{2}^{0}\left(D_{T}, S_{T}\right)}=0, \quad \lim _{m \rightarrow \infty}\left\|L_{0} u^{m}-F\right\|_{L_{2}\left(D_{T}\right)}=0 .
$$

For the solution $u \in \stackrel{0}{C}^{2}\left(\bar{D}_{T}, S_{T}^{0}, S_{T}\right)$ of the problem (3.1), (3.2) the following estimate

$$
\|u\|_{W_{2}^{0}\left(D_{T}, S_{T}\right)} \leq c(T)\|F\|_{L_{2}\left(D_{T}\right)}, \quad c(T)=\sqrt{T} \exp \frac{1}{2}\left(T+T^{2}\right),
$$

is valid. Indeed, after scalar multiplication of the both parts of the vector equation (3.1) by $2 \frac{\partial u}{\partial t}$ and integration in the domain $D_{\tau}, 0<\tau \leq T$, and simple transformations by help of the equalities (3.2) and integration by parts we receive the equality $[18],[24$, p. 116]

$$
\int_{\Omega_{\tau}}\left[\left(\frac{\partial u}{\partial t}\right)^{2}+\sum_{i=1}^{n}\left(\frac{\partial u}{\partial x_{i}}\right)^{2}\right] d x=2 \int_{D_{\tau}} F \frac{\partial u}{\partial t} d x d t
$$

where $\Omega_{\tau}:=D_{T} \cap\{t=\tau\}$. Since $S_{\tau}: t=|x|, x_{n} \geq 0, t \leq T$, then due to (3.2) we have

$$
u(x, \tau)=\int_{|x|}^{\tau} \frac{\partial}{\partial t} u(x, s) d s, \quad(x, \tau) \in \Omega_{\tau} .
$$

Squaring scalarly the both parts of the received equality, integrating it in the domain $\Omega_{\tau}$ and using the Schwartz inequality, we obtain

$$
\begin{aligned}
\int_{\Omega_{\tau}} u^{2} d x & =\int_{\Omega_{\tau}}\left(\int_{|x|}^{\tau} \frac{\partial}{\partial t} u(x, s)\right)^{2} d x \leq \int_{\Omega_{\tau}}(\tau-|x|)\left[\int_{|x|}^{\tau}\left(\frac{\partial u}{\partial t}\right)^{2} d s\right] d x \\
& \leq T \int_{\Omega_{\tau}}\left[\int_{|x|}^{\tau}\left(\frac{\partial u}{\partial t}\right)^{2} d s\right] d x=T \int_{D_{\tau}}\left(\frac{\partial u}{\partial t}\right)^{2} d x d t
\end{aligned}
$$

Let

$$
w(\tau):=\int_{\Omega_{\tau}}\left[u^{2}+\left(\frac{\partial u}{\partial t}\right)^{2}+\sum_{i=1}^{n}\left(\frac{\partial u}{\partial x_{i}}\right)^{2}\right] d x .
$$

Taking into account the inequality $2 F \frac{\partial u}{\partial t} \leq\left(\frac{\partial u}{\partial t}\right)^{2}+F^{2}$, due to (3.5) and (3.6) we have 


$$
\begin{aligned}
w(\tau) & \leq(1+T) \int_{D_{T}}\left(\frac{\partial u}{\partial t}\right)^{2} d x d t+\int_{D_{\tau}} F^{2} d x d t \\
& \leq(1+T) \int_{D_{T}}\left[u^{2}+\left(\frac{\partial u}{\partial t}\right)^{2}+\sum_{i=1}^{n}\left(\frac{\partial u}{\partial x_{i}}\right)^{2}\right] d x d t+\|F\|_{L_{2}\left(D_{T}\right)}^{2} \\
& =(1+T) \int_{0}^{\tau} w(s) d s+\|F\|_{L_{2}\left(D_{T}\right)}^{2}, \quad 0<\tau \leq T .
\end{aligned}
$$

According to the Gronwall's Lemma [25, p.13] from (3.7) it follows that

$$
w(\tau) \leq\|F\|_{L_{2}\left(D_{T}\right)}^{2} \exp (1+T) T, \quad 0<\tau \leq T
$$

By help of (3.8) we have

$$
\begin{aligned}
\|u\|_{W_{2}^{1}\left(D_{T}, S_{T}\right)}^{2} & =\int_{D_{\tau}}\left[u^{2}+\left(\frac{\partial u}{\partial t}\right)^{2}+\sum_{i=1}^{n}\left(\frac{\partial u}{\partial x_{i}}\right)^{2}\right] d x d t \\
& =\int_{0}^{T} w(\tau) d \tau \leq T\|F\|_{L_{2}\left(D_{T}\right)}^{2} \exp (1+T) T,
\end{aligned}
$$

whence it follows the estimate (3.4).

Remark 3.2. Due to (3.3) a priori estimate (3.4) is also valid for a strong generalized solution of the problem (3.1), (3.2) of the class $W_{2}^{1}$ in the domain $D_{T}$.

Since the space $C_{0}^{\infty}\left(D_{T}\right)$ of finite infinitely differentiable in the $D_{T}$ functions is dense in the $L_{2}\left(D_{T}\right)$, then for given $F=\left(F_{1}, \ldots, F_{N}\right) \in L_{2}\left(D_{T}\right)$ there exists the sequence of vector-functions $F^{m}=\left(F_{1}^{m}, \ldots, F_{N}^{m}\right) \in C_{0}^{\infty}\left(D_{T}\right)$ such that $\lim _{m \rightarrow \infty}\left\|F^{m}-F\right\|_{L_{2}\left(D_{T}\right)}=0$. For fixed $m$, extending $F^{m}$ with even manner with respect to the variable $x_{n}$ in the domain $D_{T}^{-}:=\left\{(x, t) \in \mathbb{R}^{n+1}\right.$ : $\left.x_{n}<0,|x|<t<T\right\}$ and then with zero beyond the domain $D_{T} \cup D_{T}^{-}$and leaving the same notation for it, we shall have $F^{m} \in C^{\infty}\left(\mathbb{R}_{+}^{n+1}\right)$, for which the support supp $F^{m} \subset D_{\infty} \cup D_{\infty}^{-}$, where $\mathbb{R}_{+}^{n+1}:=\mathbb{R}^{n+1} \cap\{t \geq 0\}$. Denote by $u^{m}$ the solution of the Cauchy problem:

$$
L_{0} u^{m}:=\square u^{m}=F^{m},\left.\quad u^{m}\right|_{t=0}=0,\left.\quad \frac{\partial u^{m}}{\partial t}\right|_{t=0}=0,
$$

which, as it is well-known [26, p. 192], exists, is unique and belongs to the space $C^{\infty}\left(\mathbb{R}_{+}^{n+1}\right)$. Since supp $F^{m} \subset D_{\infty} \cup D_{\infty}^{-} \subset\left\{(x, t) \in \mathbb{R}^{n+1}: t>|x|\right\},\left.u^{m}\right|_{t=0}=0$ and $\left.\frac{\partial u^{m}}{\partial t}\right|_{t=0}=0$, then taking into account geometry of the domain of dependence of the solution of linear wave equation $L_{0} u^{m}=F^{m}$, we shall have supp $u^{m} \subset\left\{(x, t) \in \mathbb{R}^{n+1}: t>|x|\right\}\left[26\right.$, p. 191] and, in particular, $\left.u^{m}\right|_{S_{T}}=0$. On the other hand the vector-function $\tilde{u}^{m}\left(x_{1}, \ldots, x_{n}, t\right)=u^{m}\left(x_{1}, \ldots,-x_{n}, t\right)$ is also a solution of the same Cauchy problem (3.9), because the vector-function $F^{m}$ is even with respect to the variable $x_{n}$. Therefore, due to the uniqueness of the solution of the Cauchy problem we have $\tilde{u}^{m}=u^{m}$, i.e. $u^{m}\left(x_{1}, \ldots,-x_{n}, t\right)=$ $u^{m}\left(x_{1}, \ldots, x_{n}, t\right)$, and thereby the vector-function $u^{m}$ is also an even function with respect the variable $x_{n}$. Whence, in turn, it follows that $\left.\frac{\partial u^{m}}{\partial x_{n}}\right|_{x_{n}=0}=0$, which with the condition $\left.u^{m}\right|_{S_{T}}=0$ means that if we leave the same notation 
for the restriction of vector-function $u^{m}$ in the domain $D_{T}$, then it is obvious that $u^{m} \in \stackrel{0}{C}^{2}\left(\bar{D}_{T}, S_{T}^{0}, S_{T}\right)$. Further, due to (3.4) and (3.9) the following inequality

$$
\lim _{m \rightarrow \infty}\left\|u^{m}-u^{k}\right\|_{W_{2}^{1}\left(D_{T}, S_{T}\right)} \leq c(T)\left\|F^{m}-F^{k}\right\|_{L_{2}\left(D_{T}\right)}
$$

is valid.

Since the sequence $\left\{F^{m}\right\}$ is fundamental in $L_{2}\left(D_{T}\right)$, then due to (3.10) the sequence $\left\{u^{m}\right\}$ is also fundamental in the complete space ${ }_{W}^{0} \underset{2}{1}\left(D_{T}, S_{T}\right)$. Therefore, there exists a vector-function $u \in \stackrel{0}{W}_{2}^{1}\left(D_{T}, S_{T}\right)$ such that $\lim _{m \rightarrow \infty}$ $\left\|u^{m}-u\right\|_{W_{2}^{1}\left(D_{T}, S_{T}\right)}=0$, and since $L_{0} u^{m}=F^{m} \rightarrow F$ in the space $L_{2}\left(D_{T}\right)$, then this vector-function according to the Remark 3.1 will be a strong generalized solution of the problem $(3.1),(3.2)$ of the class $W_{2}^{1}$ in the domain $D_{T}$. The uniqueness of this solution from the space ${ }_{W}^{0}{ }_{2}^{1}\left(D_{T}, S_{T}\right)$ follows, due to the Remark 3.2, from the a priori estimate (3.4). Therefore, for the solution $u$ of the problem $(3.1),(3.2)$ we can write $u=L_{0}^{-1} F$, where $L_{0}^{-1}:\left[L_{2}\left(D_{T}\right)\right]^{N} \rightarrow$ $\left[{ }^{0}{ }_{2}^{1}\left(D_{T}, S_{T}\right)\right]^{N}$ is a linear continuous operator with a norm admitting in view of (3.4) the following estimate

$$
\left\|L_{0}^{-1}\right\|_{\left[L_{2}\left(D_{T}\right)\right]^{N} \rightarrow\left[\stackrel{0}{W}_{2}^{1}\left(D_{T}, S_{T}\right)\right]^{N}} \leq \sqrt{T} \exp \frac{1}{2}\left(T^{2}+T\right) .
$$

Remark 3.3. Taking into account (3.11), when the condition (2.3) is fulfilled, where $0 \leq \alpha<\frac{n+1}{n-1}$ and $F \in L_{2}\left(D_{T}\right)$, due to the Remark 2.1, it is easy to see that the vector-function $u=\left(u_{1}, \ldots, u_{N}\right) \in \stackrel{0}{W} \underset{2}{1}\left(D_{T}, S_{T}\right)$ represents a strong generalized solution of the problem (2.1), (2.2) of the class $W_{2}^{1}$ in the domain $D_{T}$ if and only if $u$ is a solution of the following functional equation

$$
u=L_{0}^{-1}(-f(u)+F)
$$

in the space $\stackrel{0}{W} \underset{2}{1}\left(D_{T}, S_{T}\right)$.

Remark 3.4. Let the condition (2.3) be fulfilled and $0 \leq \alpha<\frac{n+1}{n-1}$. Rewrite the equation (3.12) in the form

$$
u=A u:=L_{0}^{-1}\left(-K_{0} u+F\right),
$$

where the operator $K_{0}:\left[\stackrel{0}{W}_{2}^{1}\left(D_{T}, S_{T}\right)\right]^{N} \rightarrow\left[L_{2}\left(D_{T}\right)\right]^{N}$ from (2.4) due to the Remark 2.1 is a continuous and compact operator. Therefore, according to $(3.11)$ and $(3.13)$ the operator $A:\left[\stackrel{0}{W} \underset{2}{1}\left(D_{T}, S_{T}\right)\right]^{N} \rightarrow\left[\stackrel{0}{W} \underset{2}{1}\left(D_{T}, S_{T}\right)\right]^{N}$ is also continuous and compact. Denote by $B\left(0, r_{0}\right):=\left\{u=\left(u_{1}, \ldots, u_{N}\right) \in\right.$ $\left.\stackrel{0}{W} \underset{2}{1}\left(D_{T}, S_{T}\right):\|u\|_{W_{2}^{0}\left(D_{T}, S_{T}\right)} \leq r_{0}\right\}$ a closed (convex) ball with radius $r_{0}>0$ and center in null element in the Hilbert space ${ }_{W}^{0} \underset{2}{1}\left(D_{T}, S_{T}\right)$. Since the operator $A$ from (3.13), acting in the space $\stackrel{0}{W}_{2}^{1}\left(D_{T}, S_{T}\right)$, is a compact continuous 
operator, then, according to the Schauder principle, for solvability of the equation (3.13) in the space ${ }_{W}^{W} \frac{1}{2}\left(D_{T}, S_{T}\right)$ it suffices to prove that the operator $A$ maps the ball $B\left(0, r_{0}\right)$ into itself for some $r_{0}>0$ [27, p. 370].

Theorem 3.1. Let $f$ satisfy the condition (2.3), where $1 \leq \alpha<\frac{n+1}{n-1} ; g=0, F \in$ $L_{2, l o c}\left(D_{\infty}\right)$ and $\left.F\right|_{D_{T}} \in L_{2}\left(D_{T}\right)$ for any $T>0$. Then the problem $(1.1),(1.2)$ is locally solvable in the class $W_{2}^{1}$, i.e. there exists a number $T_{0}=T_{0}(F)>0$ such that for any $T<T_{0}$ this problem has a strong generalized solution of the class $W_{2}^{1}$ in the domain $D_{T}$ in the sense of the Definition 2.1.

Proof. Due to Remark 3.4 it suffices to prove the existence of positive numbers $T_{0}=T_{0}(F)$ and $r_{0}=r_{0}(T, F)$ such that for $T<T_{0}$ the operator $\mathrm{A}$ from (3.13) maps the ball $B\left(0, r_{0}\right)$ into itself. For this purpose let us evaluate $\|A u\|_{W_{2}^{o}\left(D_{T}, S_{T}\right)}$ for $u \in \stackrel{0}{W} \underset{2}{1}\left(D_{T}, S_{T}\right)$. If $u=\left(u_{1}, \ldots, u_{N}\right) \in \stackrel{0}{W}{ }_{2}^{1}\left(D_{T}, S_{T}\right)$, then let us denote by $\tilde{u}$ the vector-function which represents an even extension of $u$ through planes $x_{n}=0$ and $t=T$. It is obvious that $\tilde{u} \in \stackrel{0}{W}_{2}^{1}\left(D_{T}^{*}\right):=\{v \in$ $\left.W_{2}^{1}\left(D_{T}^{*}\right):\left.v\right|_{\partial D_{T}^{*}}=0\right\}$, where $D_{T}^{*}:|x|<t<2 T-|x|$.

Using inequality $[28$, p. 258]

$$
\int_{\Omega}|v| d \Omega \leq(m e s \Omega)^{1-1 / p}\|v\|_{p, \Omega}, \quad p \geq 1,
$$

and taking into account equalities

$$
\|\tilde{u}\|_{L_{p}\left(D_{T}^{*}\right)}^{p}=4\|u\|_{L_{p}\left(D_{T}\right)}^{p}, \quad\|\tilde{u}\|_{W_{\frac{1}{2}\left(D_{T}^{*}\right)}^{*}}^{2}=4\|u\|_{W_{\frac{1}{2}}^{\prime}\left(D_{T}, S_{T}\right)}^{2},
$$

from known multiplicative inequality [21, p. 78]

$$
\begin{gathered}
\|v\|_{p, \Omega} \leq \beta\left\|\nabla_{x, t} v\right\|_{m, \Omega}^{\tilde{\alpha}}\|v\|_{r, \Omega}^{1-\tilde{\alpha}} \forall v \in \stackrel{0}{W}_{2}^{1}(\Omega), \Omega \subset \mathbb{R}^{n+1}, \\
\nabla_{x, t}=\left(\frac{\partial}{\partial x_{1}}, \ldots, \frac{\partial}{\partial x_{n}}, \frac{\partial}{\partial t}\right), \quad \tilde{\alpha}=\left(\frac{1}{r}-\frac{1}{p}\right)\left(\frac{1}{r}-\frac{1}{\tilde{m}}\right)^{-1}, \quad \tilde{m}=\frac{(n+1) m}{n+1-m}
\end{gathered}
$$

for $\Omega=D_{T}^{*} \subset \mathbb{R}^{n+1}, v=\tilde{u}, r=1, m=2$ and $1<p \leq \frac{2(n+1)}{n+1-m}$, where $\beta=$ const $>0$ does not depend on $v$ and $T$, we obtain the following inequality:

$$
\|u\|_{L_{p}\left(D_{T}\right)} \leq c_{0}\left(\text { mes } D_{T}\right)^{\frac{1}{p}+\frac{1}{n+1}-\frac{1}{2}}\|u\|_{W_{2}^{\frac{1}{2}\left(D_{T}, S_{T}\right)}} \forall u \in \stackrel{0}{W} \underset{2}{1}\left(D_{T}, S_{T}\right),
$$

where $c_{0}=$ const $>0$ does not depend on $u$ and $T$.

Since mes $D_{T}=\frac{\omega_{n}}{n+1} T^{n+1}$, where $\omega_{n}$ is the volume of a unit ball in $\mathbb{R}^{n}$, then for $p=2 \alpha$ from (3.14) we have 


$$
\|u\|_{L_{2 \alpha}\left(D_{T}\right)} \leq C_{T}\|u\|_{W_{\frac{1}{2}\left(D_{T}, S_{T}\right)}^{0}} \forall u \in \stackrel{0}{W} \underset{2}{1}\left(D_{T}, S_{T}\right),
$$

where $C_{T}=c_{0}\left(\frac{\omega_{n}}{n+1}\right)^{\alpha_{1}} T^{(n+1) \alpha_{1}}, \alpha_{1}=\frac{1}{2 \alpha}+\frac{1}{n+1}-\frac{1}{2}$.

Note that $\alpha_{1}=\frac{1}{2 \alpha}+\frac{1}{n+1}-\frac{1}{2}>0$ for $\alpha<\frac{n+1}{n-1}$, and therefore

$$
\lim _{T \rightarrow 0} C_{T}=0 \text {. }
$$

For $\left\|K_{0} u\right\|_{L_{2}\left(D_{T}\right)}$, where $u \in \stackrel{0}{W} \underset{2}{1}\left(D_{T}, S_{T}\right)$ and operator $K_{0}$ acts according to the formula (2.4), due to (2.3) and (3.15) we have the following estimate

$$
\begin{aligned}
\left\|K_{0} u\right\|_{L_{2}\left(D_{T}\right)}^{2} \leq & \int_{D_{T}}\left(M_{1}+M_{2}|u|^{\alpha}\right)^{2} d x d t \leq 2 M_{1}^{2} \text { mes } D_{T} \\
& +2 M_{2}^{2} \int_{D_{T}}|u|^{2 \alpha} d x d t=2 M_{1}^{2} \text { mes } D_{T}+2 M_{2}^{2}\|u\|_{L_{2 \alpha}\left(D_{T}\right)}^{2 \alpha} \\
\leq & 2 M_{1}^{2} \operatorname{mes}_{T}+2 M_{2}^{2} C_{T}^{2 \alpha}\|u\|_{W_{2}^{1}\left(D_{T}, S_{T}\right)}^{2 \alpha},
\end{aligned}
$$

whence we have

$$
\left\|K_{0} u\right\|_{L_{2}\left(D_{T}\right)} \leq M_{1}\left(2 \text { mes } D_{T}\right)^{\frac{1}{2}}+\sqrt{2} M_{2} C_{T}^{\alpha}\|u\|_{W_{\frac{1}{2}}\left(D_{T}, S_{T}\right)}^{\alpha} .
$$

From (3.11), (3.13) and (3.17) it follows that

$$
\begin{aligned}
\|A u\|_{W_{2}^{0}\left(D_{T}, S_{T}\right)}= & \left\|L_{0}^{-1}\left(-K_{0} u+F\right)\right\|_{W_{2}^{0}\left(D_{T}, S_{T}\right)} \\
\leq & \left.\left\|L_{0}^{-1}\right\|_{\left[L_{2}\left(D_{T}\right)\right]^{N} \rightarrow\left[W_{2}^{1}\left(D_{T}, S_{T}\right)\right.}\right]^{N}\left\|\left(-K_{0} u+F\right)\right\|_{L_{2}\left(D_{T}\right)} \\
\leq & {\left[\sqrt{T} \exp \frac{1}{2}\left(T+T^{2}\right)\right]\left(\left\|K_{0} u\right\|_{L_{2}\left(D_{T}\right)}+\|F\|_{L_{2}\left(D_{T}\right)}\right) } \\
\leq & {\left[\sqrt{T} \exp \frac{1}{2}\left(T+T^{2}\right)\right]\left(M_{1}\left(2 \text { mes } D_{T}\right)^{\frac{1}{2}}\right.} \\
& +\sqrt{2} M_{2} C_{T}^{\alpha}\|u\|_{\underset{W}{1}\left(D_{T}, S_{T}\right)}^{\alpha} \underset{W_{\frac{1}{2}\left(D_{T}, S_{T}\right)}^{\alpha}}{\alpha}+b(T) .
\end{aligned}
$$

Here

$$
\begin{gathered}
a(T)=\sqrt{2} M_{2} C_{T}^{\alpha} \sqrt{T} \exp \frac{1}{2}\left(T+T^{2}\right) \\
b(T)=\left[\sqrt{T} \exp \frac{1}{2}\left(T+T^{2}\right)\right]\left(M_{1}\left(2 m e s D_{T}\right)^{1 / 2}+\|F\|_{L_{2}\left(D_{T}\right)}\right) .
\end{gathered}
$$

For fixed $T>0$ consider equation

$$
a z^{\alpha}+b=z
$$

with respect to unknown $z \in \mathbb{R}$, where $a=a(T)$ and $b=b(T)$ are defined by (3.19) and (3.20).

First consider the case when $\alpha>1$. Simple analysis, analogous to that for $\alpha=3$ performed in the work [27, pp. 373, 374], shows that: 
1. for $b=0$, together with trivial root $z_{1=0}$, equation (3.21) has a unique positive root $z_{2}=a^{-1 /(\alpha-1)}$;

2. if $b>0$, then for $0<b<b_{0}$, where

$$
b_{0}=b_{0}(T)=\left[\alpha^{-1 /(\alpha-1)}-\alpha^{-\alpha /(\alpha-1)}\right] a^{-1 /(\alpha-1)},
$$

equation (3.21) has two positive roots $z_{1}$ and $z_{2}, 0<z_{1}<z_{2}$, and for $b=b_{0}$ these roots merge and we have one positive root $z_{1}=z_{2}=z_{0}=$ $(\alpha a)^{-1 /(\alpha-1)}$;

3. for $b>b_{0}$ equation (3.21) does not have nonnegative roots. Note that for $0<b<b_{0}$ the inequality $z_{1}<z_{0}=(\alpha a)^{-1 /(\alpha-1)}<z_{2}$ is valid.

In view of absolute continuity of the Lebesgue integral we have $\lim _{T \rightarrow 0}$ $\|F\|_{L_{2}\left(D_{T}\right)}=0$. Therefore, taking into account that mes $D_{T}=\frac{\omega_{n}}{n+1} T^{n+1}$, then from (3.20) it follows that $\lim _{T \rightarrow 0} b(T)=0$. At the same time, since $-\frac{1}{\alpha-1}<0$ for $\alpha>1$, due to (3.16) from (3.19) and (3.22) we get $\lim _{T \rightarrow 0} b_{0}(T)=\infty$. Therefore, there exists a number $T_{0}=T_{0}(F)>0$ such that for $0<T<T_{0}$ in view of (3.19)-(3.22) it will hold condition $0<b<b_{0}$, and thereby equation (3.21) will have at least one positive root, we denote it by $r_{0}=r_{0}(T, F)$.

In the case when $\alpha=1$ equation (3.21) represents a linear equation, where $\lim _{T \rightarrow 0} a(T)=0$. Therefore, for $0<T<T_{0}$, where $T_{0}=T_{0}(F)$ is a sufficiently small positive number, this equation will have a unique positive root $z(T, F)=b(1-a)^{-1}$, which we also denote by $r_{0}=r_{0}(T, F)$.

Now we will show that the operator $A$ from (3.13) maps the ball $B\left(0, r_{0}\right)$ $\subset \stackrel{0}{W} \underset{2}{1}\left(D_{T}, S_{T}\right)$ into itself. Indeed, due to (3.18) and equality $a r_{0}^{\alpha}+b=r_{0}$ for any $u \in B\left(0, r_{0}\right)$ we have

$$
\|A u\|_{W_{2}^{1}\left(D_{T}, S_{T}\right)} \leq a\|u\|_{W_{2}^{\frac{1}{2}\left(D_{T}, S_{T}\right)}}^{\alpha}+b \leq a r_{0}^{\alpha}+b=r_{0} .
$$

In view of the Remark 3.4 this proves the Theorem 3.1.

Theorem 3.2. Let $f$ satisfy the condition (2.3), where $0 \leq \alpha<1 ; g=0, F \in$ $L_{2, l o c}\left(D_{\infty}\right)$ and $\left.F\right|_{D_{T}} \in L_{2}\left(D_{T}\right)$ for any $T>0$. Then the problem $(1.1),(1.2)$ is globally solvable in the class $W_{2}^{1}$, i.e. for any $T>0$ this problem has a strong generalized solution of the class $W_{2}^{1}$ in the domain $D_{T}$ in the sense of the Definition 2.1 .

Proof. According to the Remark 3.4 for the proof of the Theorem 3.2 it suffices to show that for any $T>0$ there exists a number $r_{0}=r_{0}(T, F)>0$ such that the operator $A$ from (3.13) maps the ball $B\left(0, r_{0}\right) \subset \stackrel{0}{W}{ }_{2}^{1}\left(D_{T}, S_{T}\right)$ into itself. Let $1 / 2<\alpha<1$, then, since $2 \alpha>1$ the inequality (3.15) is valid, and therefore, the estimate (3.18) is also valid. For fixed $T>0$, because $\alpha<1$ there exists a number $r_{0}=r_{0}(T, F)>0$ such that

$$
a(T) s^{\alpha}+b(T) \leq r_{0} \quad \forall s \in\left[0, r_{0}\right] .
$$

Indeed, the function $\frac{\lambda(s)}{s}$, where $\lambda(s)=a(T) s^{\alpha}+b(T)$, is a continuous decreasing function and $\lim _{s \rightarrow+0} \frac{\lambda(s)}{s}=+\infty, \lim _{s \rightarrow+\infty} \frac{\lambda(s)}{s}=0$. Therefore there exists a number $s=r_{0}(T, F)>0$ such that $\left.\frac{\lambda(s)}{s}\right|_{s=r_{0}}=1$. Whence, since the function 
$\lambda(s)$ for $s \geq 0$ is a monotonic increasing function, (3.24) follows immediately. Now, in view of (3.18) and (3.24), for any $u \in B\left(0, r_{0}\right)$ it is valid the inequality $(3.23)$, i.e. $A\left(B\left(0, r_{0}\right)\right) \subset B\left(0, r_{0}\right)$.

The case $0 \leq \alpha \leq \frac{1}{2}$ can be reduced to the previous case $\frac{1}{2}<\alpha<1$ because the vector-function $f$, satisfying the condition (2.3) for $0 \leq \alpha \leq \frac{1}{2}$, satisfies the same condition (2.3) for a certain fixed $\alpha=\alpha_{1} \in\left(\frac{1}{2}, 1\right)$ with other positive constants $M_{1}$ and $M_{2}$ (it is easy to see that $M_{1}+M_{2}|u|^{\alpha} \leq$ $\left.\left(M_{1}+M_{2}\right)+M_{2}|u|^{\alpha_{1}} \forall u \in \mathbb{R}, \alpha<\alpha_{1}\right)$. This proves the Theorem 3.2 completely.

Remark 3.5. The global solvability of the problem (1.1), (1.2) in the Theorem 3.2 is proved for the case when the function $f$ satisfies the condition (2.3), where $0 \leq \alpha<1$. In the case $1 \leq \alpha<\frac{n+1}{n-1}$ in the Theorem 3.1 it is proved the local solvability of this problem, although in this case for additional conditions posed on $f$, the problem (1.1), (1.2) will be globally solvable as it is shown in the following theorem.

Theorem 3.3. Let $f$ satisfy the condition (2.3), where $1 \leq \alpha<\frac{n+1}{n-1}$, and $f=$ $\nabla G$, i.e. $f_{i}(u)=\frac{\partial}{\partial u_{i}} G(u), u \in \mathbb{R}^{N}, i=1, \ldots, N$, where $G=G(u) \in C^{1}\left(\mathbb{R}^{N}\right)$ is a scalar function satisfying conditions $G(0)=0$ and $G(u) \geq 0 \forall u \in \mathbb{R}^{N}$. Let $g=0, F \in L_{2, l o c}\left(D_{\infty}\right)$ and $\left.F\right|_{D_{T}} \in L_{2}\left(D_{T}\right)$ for any $T>0$. Then the problem (1.1), (1.2) is globally solvable in the class $W_{2}^{1}$, i.e. for any $T>0$ this problem has a strong generalized solution of the class $W_{2}^{1}$ in the domain $D_{T}$ in the sense of the Definition 2.1 .

Proof. First let us show that for any fixed $T>0$, when the conditions of the Theorem 3.3 are fulfilled, for a strong generalized solution $u$ of the problem (1.1), (1.2) of the class $W_{2}^{1}$ in the domain $D_{T}$ the a priori estimate (3.4) is valid. Indeed, due to the Definition 2.1 there exists a sequence of of the vector-functions $u^{m} \in \stackrel{0}{C}^{2}\left(\bar{D}_{T}, S_{T}^{0}, S_{T}\right)$ such that

$$
\lim _{m \rightarrow \infty}\left\|u^{m}-u\right\|_{W_{2}^{1}\left(D_{T}\right)}=0, \quad \lim _{m \rightarrow \infty}\left\|L u^{m}-F\right\|_{L_{2}\left(D_{T}\right)}=0 .
$$

Let

$$
F^{m}:=L u^{m}
$$

then due to the equality (3.5) we have

$$
\int_{\Omega_{\tau}}\left[\left(\frac{\partial u^{m}}{\partial t}\right)^{2}+\sum_{i=1}^{n}\left(\frac{\partial u^{m}}{\partial x_{i}}\right)^{2}\right] d x=2 \int_{D_{\tau}}\left(F^{m}-f\left(u^{m}\right)\right) \frac{\partial u^{m}}{\partial t} d x d t
$$

Since $f=\nabla G$, then $f\left(u^{m}\right) \frac{\partial u^{m}}{\partial t}=\frac{\partial}{\partial t} G\left(u^{m}\right)$ and taking into account that $\left.u^{m}\right|_{S_{T}}=0,\left.\nu_{n+1}\right|_{S_{\tau}^{0}}=0,\left.\nu_{n+1}\right|_{\Omega_{\tau}}=1, G(0)=0$, by integration by parts we have 


$$
\begin{aligned}
\int_{D_{\tau}} f\left(u^{m}\right) \frac{\partial u^{m}}{\partial t} d x d t & =\int_{D_{\tau}} \frac{\partial}{\partial t} G\left(u^{m}\right) d x d t=\int_{\partial D_{\tau}} G\left(u^{m}\right) \nu_{n+1} d s \\
& =\int_{S_{\tau}^{0} \cup S_{\tau} \cup \Omega_{\tau}} G\left(u^{m}\right) \nu_{n+1} d s=\int_{\Omega_{\tau}} G\left(u^{m}\right) d x .
\end{aligned}
$$

In view of (3.28) and $G \geq 0$ from (3.27) we have

$$
\begin{aligned}
\int_{\Omega_{\tau}}\left[\left(\frac{\partial u^{m}}{\partial t}\right)^{2}+\sum_{i=1}^{n}\left(\frac{\partial u^{m}}{\partial x_{i}}\right)^{2}\right] d x= & 2 \int_{D_{\tau}} F^{m} \frac{\partial u^{m}}{\partial t} d x d t \\
& -2 \int_{\Omega_{\tau}} G\left(u^{m}\right) d x \leq 2 \int_{D_{\tau}} F^{m} \frac{\partial u^{m}}{\partial t} d x d t .
\end{aligned}
$$

Using the same reasonings as those used for receiving the estimate (3.4), from (3.29) we get the following inequality

$$
\left\|u^{m}\right\|_{W_{\frac{1}{2}\left(D_{T}, S_{T}\right)}^{0}} \leq c(T)\left\|F^{m}\right\|_{L_{2}\left(D_{T}\right)}, c(T)=\sqrt{T} \exp \frac{1}{2}\left(T+T^{2}\right),
$$

whence, due to (3.25) and (3.26), it follows (3.4).

According to the Remarks 3.3 and 3.4, at fulfilment of the conditions of the Theorem 3.3, the vector-function $u \in \stackrel{0}{W} \underset{2}{1}\left(D_{T}, S_{T}\right)$ represents a strong generalized solution of the problem (1.1), (1.2) of the class $W_{2}^{1}$ then and only then, when $u$ represents a solution to the functional equation $u=A u$ from (3.13) in the space $\stackrel{0}{W}_{2}^{1}\left(D_{T}, S_{T}\right)$, where the operator $A:\left[{ }_{W}^{0} \frac{1}{2}\left(D_{T}, S_{T}\right)\right]^{N} \rightarrow$ $\left[{ }_{W}^{\infty} \frac{1}{2}\left(D_{T}, S_{T}\right)\right]^{N}$ is continuous and compact. At the same time, as it was shown above, for any $\mu \in[0,1]$ and for any solution of the equation $u=\mu A u$ with the parameter $\mu$ in the space $\stackrel{0}{W}_{2}^{1}\left(D_{T}, S_{T}\right)$ it is valid the following a priori estimate $\|u\|_{W_{\frac{1}{2}\left(D_{T}, S_{T}\right)}} \leq \mu c(T)\|F\|_{L_{2}\left(D_{T}\right)} \leq c(T)\|F\|_{L_{2}\left(D_{T}\right)}$ with positive constant $c(T)$, not depending on $u, \mu$ and $F$. Therefore, according to the Leray-Schauder theorem [27, p. 375] the equation (3.13), and therefore the problem (1.1), (1.2) has at least one strong generalized solution of the class $W_{2}^{1}$ in the domain $D_{T}$ for any $T>0$. The Theorem 3.3 is proved.

\section{The uniqueness and existence of a global solution of the problem (1.1), (1.2) in the class $W_{2}^{1}$}

Below we impose on the nonlinear vector-function $f=\left(f_{1}, \ldots, f_{N}\right)$ from (1.1) additional requirements

$$
f \in C^{1}\left(\mathbb{R}^{N}\right), \quad\left|\frac{\partial f_{i}(u)}{\partial u_{j}}\right| \leq M_{3}+M_{4}|u|^{\gamma} \forall u \in \mathbb{R}^{n}, \quad 1 \leq i, j \leq N,
$$

where $M_{3}, M_{4}, \gamma=$ const $\geq 0$. For simplicity we assume that the vector-function $g=0$ in the boundary condition (1.2). 
Remark 4.1. It is obvious that from (4.1) it follows the condition (2.3) for $\gamma=\alpha-1$, and in the case $\gamma<\frac{2}{n-1}$ we have $1 \leq \alpha=\gamma+1<\frac{n+1}{n-1}$.

Theorem 4.1. Let the condition (4.1) be fulfilled, where $0 \leq \gamma<\frac{2}{n-1} ; F \in$ $L_{2}\left(D_{T}\right)$ and $g=0$. Then the problem (1.1), (1.2) cannot have more than one strong generalized solution of the class $W_{2}^{1}$ in the domain $D_{T}$ in the sense of the Definition 2.1.

Proof. Let $F \in L_{2}\left(D_{T}\right), g=0$, and assume that the problem (1.1), (1.2) has two strong generalized solutions $u^{1}$ and $u^{2}$ of the class $W_{2}^{1}$ in the domain $D_{T}$ in the sense of Definition 2.1, i.e. there exist two sequences of vector-functions $u^{i m} \in \stackrel{0}{C}^{2}\left(\bar{D}_{T}, S_{T}^{0}, S_{T}\right), i=1,2 ; m=1,2, \ldots$, such that

$\lim _{m \rightarrow \infty}\left\|u^{i m}-u^{i}\right\|_{W_{2}^{0}\left(D_{T}, S_{T}\right)}=0, \quad \lim _{m \rightarrow \infty}\left\|L u^{i m}-F\right\|_{L_{2}\left(D_{T}\right)}=0, \quad i=1,2$.

Let

$$
w=u^{2}-u^{1}, \quad w^{m}=u^{2 m}-u^{1 m}, \quad F^{m}=L u^{2 m}-L u^{1 m} .
$$

In view of (4.2) and (4.3) we have

$$
\lim _{m \rightarrow \infty}\left\|w^{m}-w\right\|_{W_{2}^{0}\left(D_{T}, S_{T}\right)}=0, \lim _{m \rightarrow \infty}\left\|F^{m}\right\|_{L_{2}\left(D_{T}\right)}=0 .
$$

In accordance with $(4.3)$ consider the vector-function $w^{m} \in \stackrel{0}{C}^{2}\left(\bar{D}_{T}, S_{T}^{0}\right.$, $\left.S_{T}\right)$ as a solution of the following problem

$$
\begin{gathered}
\square w^{m}=-\left[f\left(u^{2 m}\right)-f\left(u^{1 m}\right)\right]+F^{m}, \\
\left.\frac{\partial w^{m}}{\partial x_{n}}\right|_{S_{T}^{0}}=0,\left.w^{m}\right|_{S_{T}}=0 .
\end{gathered}
$$

From (4.5), (4.6) and in view of the equality (3.5) it follows

$$
\begin{gathered}
\int_{\Omega_{\tau}}\left[\left(\frac{\partial w^{m}}{\partial t}\right)^{2}+\sum_{i=1}^{n}\left(\frac{\partial w^{m}}{\partial x_{i}}\right)^{2}\right] d x=2 \int_{D_{\tau}} F^{m} \frac{\partial w^{m}}{\partial t} d x d t \\
-2 \int_{D_{\tau}}\left[f\left(u^{2 m}\right)-f\left(u^{1 m}\right)\right] \frac{\partial w^{m}}{\partial t} d x d t, \quad 0<\tau \leq T .
\end{gathered}
$$

Taking into account the equality

$$
f_{i}\left(u^{2 m}\right)-f_{i}\left(u^{1 m}\right)=\sum_{j=1}^{N} \int_{0}^{1} \frac{\partial}{\partial u_{j}} f_{i}\left(u^{1 m}+s\left(u^{2 m}-u^{1 m}\right)\right) d s\left(u_{j}^{2 m}-u_{j}^{1 m}\right),
$$

we receive

$$
\begin{aligned}
& {\left[f\left(u^{2 m}\right)-f\left(u^{1 m}\right)\right] \frac{\partial w^{m}}{\partial t}} \\
& \quad=\sum_{i, j=1}^{N}\left[\int_{0}^{1} \frac{\partial}{\partial u_{j}} f_{i}\left(u^{1 m}+s\left(u^{2 m}-u^{1 m}\right)\right) d s\right]\left(u_{j}^{2 m}-u_{j}^{1 m}\right) \frac{\partial w_{i}^{m}}{\partial t} .
\end{aligned}
$$


From (4.1) and obvious inequality $\left|d_{1}+d_{2}\right|^{\gamma} \leq 2^{\gamma} \max \left(\left|d_{1}\right|^{\gamma},\left|d_{2}\right|^{\gamma}\right) \leq$ $2^{\gamma}\left(\left|d_{1}\right|^{\gamma}+\left|d_{2}\right|^{\gamma}\right)$ for $\gamma \geq 0, d_{i} \in \mathbb{R}$, we have

$$
\begin{aligned}
\left|\int_{0}^{1} \frac{\partial}{\partial u_{j}} f_{i}\left(u^{1 m}+s\left(u^{2 m}-u^{1 m}\right)\right) d s\right| & \leq \int_{0}^{1}\left[M_{3}+M_{4}\left|(1-s) u^{1 m}+s u^{2 m}\right|^{\gamma}\right] d s \\
& \leq M_{3}+2^{\gamma} M_{4}\left(\left|u^{1 m}\right|^{\gamma}+\left|u^{2 m}\right|^{\gamma}\right) .
\end{aligned}
$$

From (4.8) and (4.9), taking into account (4.3), it follows

$$
\begin{aligned}
\left|\left[f\left(u^{2 m}\right)-f\left(u^{1 m}\right)\right] \frac{\partial w^{m}}{\partial t}\right| \leq & \sum_{i, j=1}^{N}\left[M_{3}+2^{\gamma} M_{4}\left(\left|u^{1 m}\right|^{\gamma}+\left|u^{2 m}\right|^{\gamma}\right)\right]\left|w_{j}^{m}\right|\left|\frac{\partial w_{i}^{m}}{\partial t}\right| \\
\leq & N^{2}\left[M_{3}+2^{\gamma} M_{4}\left(\left|u^{1 m}\right|^{\gamma}+\left|u^{2 m}\right|^{\gamma}\right)\right]\left|w^{m}\right|\left|\frac{\partial w^{m}}{\partial t}\right| \\
\leq & \frac{1}{2} N^{2} M_{3}\left[\left(w^{m}\right)^{2}+\left(\frac{\partial w^{m}}{\partial t}\right)^{2}\right] \\
& +2^{\gamma} N^{2} M_{4}\left(\left|u^{1 m}\right|^{\gamma}+\left|u^{2 m}\right|^{\gamma}\right)\left|w^{m}\right|\left|\frac{\partial w^{m}}{\partial t}\right| .
\end{aligned}
$$

Due to (4.7) and (4.10) we have

$$
\begin{aligned}
& \int_{\Omega_{\tau}}\left[\left(\frac{\partial w^{m}}{\partial t}\right)^{2}+\sum_{i=1}^{n}\left(\frac{\partial w^{m}}{\partial x_{i}}\right)^{2}\right] d x \leq \int_{D_{\tau}}\left[\left(\frac{\partial w^{m}}{\partial t}\right)^{2}+\left(F^{m}\right)^{2}\right] d x d t \\
& +N^{2} M_{3} \int_{D_{\tau}}\left[\left(w^{m}\right)^{2}+\left(\frac{\partial w^{m}}{\partial t}\right)^{2}\right] d x d t \\
& +2^{\gamma+1} N^{2} M_{4} \int_{D_{T}}\left(\left|u^{1 m}\right|^{\gamma}+\left|u^{2 m}\right|^{\gamma}\right)\left|w^{m}\right|\left|\frac{\partial w^{m}}{\partial t}\right| d x d t
\end{aligned}
$$

The latter integral in the right side of (4.11) can be estimated by Holder's inequality

$$
\begin{aligned}
& \int_{D_{T}}\left(\left|u^{1 m}\right|^{\gamma}+\left|u^{2 m}\right|^{\gamma}\right)\left|w^{m}\right|\left|\frac{\partial w^{m}}{\partial t}\right| d x d t \\
& \quad \leq\left(||\left|u^{1 m}\right|^{\gamma}\left\|_{L_{n+1}\left(D_{T}\right)}+\right\|\left|u^{2 m}\right|^{\gamma} \|_{L_{n+1}\left(D_{T}\right)}\right)\left\|w^{m}\right\|_{L_{p}\left(D_{\tau}\right)}\left\|\frac{\partial w^{m}}{\partial t}\right\|_{L_{2}\left(D_{\tau}\right)} .
\end{aligned}
$$

Here $\frac{1}{n+1}+\frac{1}{p}+\frac{1}{2}=1$, i.e. for

$$
p=\frac{2(n+1)}{n-1} .
$$

In view of (3.14) for $q \leq \frac{2(n+1)}{n-1}$ we have

$$
\|v\|_{L_{q}\left(D_{T}\right)} \leq C_{q}(T)\|v\|_{W_{2}^{\prime}\left(D_{T}, S_{T}\right)} \forall v \in \stackrel{0}{W} \underset{2}{1}\left(D_{\tau}, S_{\tau}\right), 0<\tau \leq T
$$

with positive constant $C_{q}(T)$, not depending on $v \in \stackrel{0}{W}{ }_{2}^{1}\left(D_{\tau}, S_{\tau}\right)$ and $\tau \in$ $[0, T]$. 
According to the theorem $\gamma<\frac{2}{n-1}$ and, therefore, $\gamma(n+1)<\frac{2(n+1)}{n-1}$. Thus, from (4.13), (4.14) we receive

$$
\begin{gathered}
\left\|\left|u^{i m}\right|^{\gamma}\right\|_{L_{n+1}\left(D_{T}\right)}=\left\|u^{i m}\right\|_{L_{\gamma(n+1)}\left(D_{T}\right)}^{\gamma} \leq C_{\gamma(n+1)}^{\gamma}(T)\left\|u^{i m}\right\|_{W_{\frac{1}{2}\left(D_{T}, S_{T}\right)}^{\gamma}}^{\gamma}, \\
i=1,2 ; m \geq 1 \\
\qquad w^{m}\left\|_{L_{p}\left(D_{\tau}\right)} \leq C_{p}(T)\right\| w^{m} \|_{W_{2}^{1}\left(D_{\tau}\right)}, m \geq 1 .
\end{gathered}
$$

In view of the first equality from (4.2) there exists natural number $m_{0}$ such that for $m \geq m_{0}$ we shall have

$$
\left\|u^{i m}\right\|_{W_{2}^{1}\left(D_{T}, S_{T}\right)}^{\gamma} \leq\left\|u^{i}\right\|_{W_{2}^{1}\left(D_{T}, S_{T}\right)}^{\gamma}+1, \quad i=1,2 ; m \geq m_{0} .
$$

In view of these inequalities from (4.12)-(4.16) it follows that

$$
\begin{aligned}
2^{\gamma+1} N^{2} M_{4} \int_{D_{\tau}}\left(\left|u^{1 m}\right|^{\gamma}+\left|u^{2 m}\right|^{\gamma}\right)\left|w^{m}\right|\left|\frac{\partial w^{m}}{\partial t}\right| d x d t \\
\leq 2^{\gamma+1} N^{2} M_{4} C_{\gamma(n+1)}^{\gamma}(T)\left(\left\|u^{1}\right\|_{W_{2}^{1}\left(D_{T}, S_{T}\right)}^{\gamma}\right. \\
\left.\quad+\left\|u^{2}\right\|_{W_{2}^{1}\left(D_{T}, S_{T}\right)}^{\gamma}+2\right) C_{p}(T)\left\|w^{m}\right\|_{W_{2}^{0}\left(D_{\tau}, S_{\tau}\right)}\left\|\frac{\partial w^{m}}{\partial t}\right\|_{L_{2}\left(D_{\tau}\right)} \\
\leq M_{5}\left(\left\|w^{m}\right\|_{W_{2}^{1}\left(D_{\tau}\right)}^{2}+\left\|\frac{\partial w^{m}}{\partial t}\right\|_{L_{2}\left(D_{\tau}\right)}^{2} \leq 2 M_{5}\left\|w^{m}\right\|_{W_{2}^{1}\left(D_{\tau}\right)}^{2}\right. \\
=2 M_{5} \int_{D_{\tau}}\left[\left(w^{m}\right)^{2}+\left(\frac{\partial w^{m}}{\partial t}\right)^{2}+\sum_{i=1}^{n}\left(\frac{\partial w^{m}}{\partial x_{i}}\right)^{2}\right] d x d t
\end{aligned}
$$

where $M_{5}=2^{\gamma} N^{2} M_{4} C_{\gamma(n+1)}^{\gamma}(T)\left(\left\|u^{1}\right\|_{W_{2}^{1}\left(D_{T}, S_{T}\right)}^{\gamma}+\left\|u^{2}\right\|_{W_{2}^{1}\left(D_{T}, S_{T}\right)}^{\gamma}+2\right) C_{p}(T)$.

Due to (4.17) from (4.11) we have

$$
\begin{aligned}
& \int_{\Omega_{\tau}}\left[\left(\frac{\partial w^{m}}{\partial t}\right)^{2}+\sum_{i=1}^{n}\left(\frac{\partial w^{m}}{\partial x_{i}}\right)^{2}\right] d x \leq M_{6} \int_{D_{\tau}}\left[\left(w^{m}\right)^{2}+\left(\frac{\partial w^{m}}{\partial t}\right)^{2}\right. \\
& \left.+\sum_{i=1}^{n}\left(\frac{\partial w^{m}}{\partial x_{i}}\right)^{2}\right] d x d t+\int_{D_{T}}\left(F^{m}\right)^{2} d x d t, \quad 0<\tau \leq T
\end{aligned}
$$

where $M_{6}=1+M_{3} N^{2}+2 M_{5}$.

Note, that the inequality (3.6) is valid for $w^{m}$ too, and, therefore,

$$
\begin{aligned}
& \int_{\Omega_{\tau}}\left(w^{m}\right)^{2} d x \leq T \int_{D_{\tau}}\left(\frac{\partial w^{m}}{\partial t}\right)^{2} d x d t \\
& \quad \leq T \int_{D_{\tau}}\left[\left(w^{m}\right)^{2}+\left(\frac{\partial w^{m}}{\partial t}\right)^{2}+\sum_{i=1}^{n}\left(\frac{\partial w^{m}}{\partial x_{i}}\right)^{2}\right] d x d t
\end{aligned}
$$

Putting

$$
\lambda_{m}(\tau):=\int_{\Omega_{\tau}}\left[\left(w^{m}\right)^{2}+\left(\frac{\partial w^{m}}{\partial t}\right)^{2}+\sum_{i=1}^{n}\left(\frac{\partial w^{m}}{\partial x_{i}}\right)^{2}\right] d x
$$


and adding (4.18) to (4.19), we receive

$$
\lambda_{m}(\tau) \leq\left(M_{6}+T\right) \int_{0}^{\tau} \lambda_{m}(s) d s+\left\|F^{m}\right\|_{L_{2}\left(D_{T}\right)}^{2} .
$$

Whence, by the Gronwall's lemma [25, p. 13], it follows that

$$
\lambda_{m}(\tau) \leq\left\|F^{m}\right\|_{L_{2}\left(D_{T}\right)}^{2} \exp \left(M_{6}+T\right) \tau .
$$

From (4.20) and (4.21) we have

$$
\left\|w^{m}\right\|_{W_{2}^{1}\left(D_{T}\right)}^{2}=\int_{0}^{T} \lambda_{m}(\tau) d \tau \leq T\left\|F^{m}\right\|_{L_{2}\left(D_{T}\right)}^{2} \exp \left(M_{6}+T\right) T .
$$

In view of (4.3), (4.4) from (4.22) it follows that

$$
\begin{aligned}
\|w\|_{W_{2}^{1}\left(D_{T}\right)} & =\lim _{m \rightarrow \infty}\left\|w-w^{m}+w^{m}\right\|_{W_{2}^{1}\left(D_{T}\right)} \\
& \leq \lim _{m \rightarrow \infty}\left\|w-w^{m}\right\|_{W_{2}^{1}\left(D_{T}\right)}+\lim _{m \rightarrow \infty}\left\|w^{m}\right\|_{W_{2}^{1}\left(D_{T}\right)} \\
& =\lim _{m \rightarrow \infty}\left\|w-w^{m}\right\|_{W_{2}^{1}\left(D_{T}\right)}=\lim _{m \rightarrow \infty}\left\|w-w^{m}\right\|_{W_{2}^{1}\left(D_{T}, S_{T}\right)}=0 .
\end{aligned}
$$

Therefore $w=u_{2}-u_{1}=0$, i.e. $u_{2}=u_{1}$. The Theorem 4.1 is proved.

From the Theorems 3.2, 3.3, 4.1 and the Remark 4.1 it follows the next theorem of existence and uniqueness.

Theorem 4.2. Let the vector-function $f$ satisfy the condition (4.1), where $0 \leq$ $\gamma<\frac{2}{n-1}$, and either $f$ satisfy the condition (2.3) for $\alpha<1$ or $f=\nabla G$, where $G \in C^{1}\left(\mathbb{R}^{N}\right), G(0)=0$ and $G(u) \geq 0 \forall u \in \mathbb{R}^{N}$. Then for any $F \in L_{2}\left(D_{T}\right)$ and $g=0$ the problem (1.1), (1.2) has unique strong generalized solution $u \in$ $\stackrel{0}{W}_{2}^{1}\left(D_{T}, S_{T}\right)$ of the class $W_{2}^{1}$ in the domain $D_{T}$ in the sense of the Definition 2.1. The following theorem on existence of global solution of this problem follows from the Theorem 4.2 .

Theorem 4.3. Let the vector-function $f$ satisfy the condition (4.1), where $0 \leq$ $\gamma<\frac{2}{n-1}$, and either $f$ satisfy the condition (2.3) for $\alpha<1$ or $f=\nabla G$, where $G \in C^{1}\left(\mathbb{R}^{N}\right), G(0)=0$ and $G(u) \geq 0 \forall u \in \mathbb{R}^{N}$. Let $g=0, F \in L_{2, l o c}\left(D_{\infty}\right)$ and $\left.F\right|_{D_{T}} \in L_{2}\left(D_{T}\right)$ for any $T>0$. Then the problem (1.1), (1.2) has unique global strong generalized solution $u \in \stackrel{0}{W} \underset{2, \text { loc }}{1}\left(D_{\infty}, S_{\infty}\right)$ of the class $W_{2}^{1}$ in the domain $D_{\infty}$ in the sense of the Definition 2.1 .

Proof. According to Theorem 4.2 when the conditions of Theorem 4.3 are fulfilled for $T=k$, where $k$ is a natural number, there exists unique strong generalized solution $u^{k} \in \stackrel{0}{W}_{2}^{1}\left(D_{T}, S_{T}\right)$ of the problem $(1.1),(1.2)$ of the class $W_{2}^{1}$ in the domain $D_{T=k}$ in the sense of Definition 2.1. Since $\left.u^{k+1}\right|_{D_{T=k}}$ is also a strong generalized solution of the problem (1.1), (1.2) of the class $W_{2}^{1}$ in the domain $D_{T=k}$, then in view of Theorem 4.2 we have $u^{k}=\left.u^{k+1}\right|_{D_{T=k}}$. Thus one can construct unique global generalized solution $u \in \stackrel{0}{W} \underset{2, l o c}{1}\left(D_{\infty}, S_{\infty}\right)$ of the problem (1.1), (1.2) of the class $W_{2}^{1}$ in the domain $D_{\infty}$ in the sense of the Definition 2.4 in the following way: 


$$
u(x, t)=u^{k}(x, t), \quad(x, t) \in D_{\infty}, \quad k=[t]+1,
$$

where $[t]$ is an integer part of the number $t$. The Theorem 4.3 is proved.

\section{The cases of absence of a global solution of the problem (1.1), (1.2) of the class $W_{2}^{1}$}

Theorem 5.1. Let the vector-function $f=\left(f_{1}, \ldots, f_{N}\right)$ satisfy the condition (2.3), where $1<\alpha<\frac{n+1}{n-1}$, and there exist numbers $l_{1}, \ldots, l_{N}, \sum_{i=1}^{N}\left|l_{i}\right| \neq 0$, such that

$$
\sum_{i=1}^{N} l_{i} f_{i}(u) \leq c_{0}-c_{1}\left|\sum_{i=1}^{N} l_{i} u_{i}\right|^{\beta} \forall u \in \mathbb{R}^{N}, 1<\beta=\text { const }<\frac{n+1}{n-1},
$$

where $c_{0}, c_{1}=$ const, $c_{1}>0$. Let $F \in L_{2, l o c}\left(D_{\infty}\right), g \in W_{2, l o c}^{1}\left(S_{\infty}\right)$ and $\left.F\right|_{D_{T}} \in$ $L_{2}\left(D_{T}\right),\left.g\right|_{S_{T}} \in W_{2}^{1}\left(S_{T}\right)$ for any $T>0$. Let at least one of functions $F_{0}=$ $\sum_{i=1}^{N} l_{i} F_{i}-c_{0}$ or $\left.\frac{\partial g_{0}}{\partial \aleph}\right|_{S_{\infty}}$, where $g_{0}=\sum_{i=1}^{N} l_{i} g_{i}$, is nontrivial (i.e. differs from zero on a subset with positive measure in $D_{\infty}$ or $S_{\infty}$, respectively). Then if

$$
g_{0} \geq 0,\left.\frac{\partial g_{0}}{\partial \aleph}\right|_{S_{\infty}} \leq 0,\left.F_{0}\right|_{D_{\infty}} \geq 0,
$$

there exists finite positive number $T_{0}=T_{0}(F, g)$ such that for $T>T_{0}$ the problem (1.1), (1.2) does not have a strong generalized solution of the class $W_{2}^{1}$ in the domain $D_{T}$ in the sense of the Definition 2.1. Here $\frac{\partial}{\partial \aleph}$ is a derivative with respect to the conormal to $S_{\infty}$, i.e. $\frac{\partial}{\partial \aleph}=\nu_{n+1} \frac{\partial}{\partial t}-\sum_{i=1}^{N} \nu_{i} \frac{\partial}{\partial x_{i}}$, where $\nu=\left(\nu_{1}, \ldots, \nu_{n}, \nu_{n+1}\right)$ is a unit vector of the outer normal to $\partial D_{\infty}=S_{\infty}$, which is an inner differential operator on the characteristic manifold $S_{\infty}$.

Proof. Let $G_{T}:|x|<t<T, G_{T}^{-}=G_{T} \cap\left\{x_{n}<0\right\}, S_{T}^{-}: t=|x|, x_{n} \leq 0, t \leq T$. It is obvious that $D_{T}=G_{T}^{+}: G_{T} \cap\left\{x_{n}>0\right\}$ and $G_{T}=G_{T}^{-} \cup\left(S_{T}^{0} \backslash \partial S_{T}^{0}\right) \cup$ $G_{T}^{+}$, where $S_{T}^{0}=\partial D_{T} \cap\left\{x_{n}=0\right\}$. Let $u=\left(u_{1}, \ldots, u_{N}\right)$ represents a strong generalized solution of the problem (1.1), (1.2) of the class $W_{2}^{1}$ in the domain $D_{T}$ in the sense of the Definition 2.1. Let us extend the vector-functions $u, F$ and $g$ evenly with respect to variable $x_{n}$ in $G_{T}^{-}$and $S_{T}^{-}$, respectively. For simplicity we leave the same notations $u, F$ and $g$ to the extended functions defined in $G_{T}$ and $S_{T}^{-} \cup S_{T}$. Let us show that the function $u=\left(u_{1}, \ldots, u_{N}\right)$, defined in the domain $G_{T}$, satisfies the following integral equality

$$
\begin{aligned}
& \int_{G_{T}}\left[-u_{t} w_{t}+\nabla u \nabla w\right] d x d t=-\int_{G_{T}} f(u) w d x d t \\
& \quad+\int_{G_{T}} F w d x d t-\int_{S_{T}^{-} \cup S_{T}} \frac{\partial g}{\partial \aleph} w d s
\end{aligned}
$$

for any vector-function $w=\left(w_{1}, \ldots, w_{N}\right) \in W_{2}^{1}\left(G_{T}\right)$ such that $\left.w\right|_{t=T}=0$ in the sense of the trace theory. Indeed, if $w \in W_{2}^{1}\left(G_{T}\right)$ and $\left.w\right|_{t=T}=0$, then it is obvious that $\left.w\right|_{D_{T}} \in W_{2}^{1}\left(D_{T}\right)$ and $\tilde{w} \in W_{2}^{1}\left(D_{T}\right)$, where by definition $\tilde{w}\left(x_{1}, \ldots, x_{n}, t\right)=w\left(x_{1}, \ldots,-x_{n}, t\right),\left(x_{1}, \ldots, x_{n}, t\right) \in D_{T}$, and $\left.\tilde{w}\right|_{t=T}=0$. 
Therefore, according to the equality (2.6) from the Remark 2.2, for $\varphi=w$ and $\varphi=\tilde{w}$ we have

$$
\begin{aligned}
\int_{D_{T}}\left[-u_{t} w_{t}+\nabla u \nabla w\right] d x d t= & -\int_{D_{T}} f(u) w d x d t \\
& +\int_{D_{T}} F w d x d t-\int_{S_{T}} \frac{\partial g}{\partial \aleph} w d s
\end{aligned}
$$

and

$$
\begin{aligned}
\int_{D_{T}}\left[-u_{t} \tilde{w}_{t}+\nabla u \nabla \tilde{w}\right] d x d t= & -\int_{D_{T}} f(u) \tilde{w} d x d t \\
& +\int_{D_{T}} F \tilde{w} d x d t-\int_{S_{T}} \frac{\partial g}{\partial \aleph} \tilde{w} d s
\end{aligned}
$$

respectively. Since $u, F$ and $g$ are even vector-functions with respect to variable $x_{n}$ and $\tilde{w}\left(x_{1}, \ldots, x_{n}, t\right)=w\left(x_{1}, \ldots,-x_{n}, t\right),\left(x_{1}, \ldots, x_{n}, t\right) \in D_{T}$, then we have

$$
\begin{gathered}
\int_{D_{T}}\left[-u_{t} \tilde{w}_{t}+\nabla u \nabla \tilde{w}\right] d x d t=\int_{G_{T}^{-}}\left[-u_{t} w_{t}+\nabla u \nabla w\right] d x d t \\
-\int_{D_{T}} f(u) \tilde{w} d x d t+\int_{D_{T}} F \tilde{w} d x d t-\int_{S_{T}} \frac{\partial g}{\partial \aleph} \tilde{w} d s \\
=-\int_{G_{T}^{-}} f(u) w d x d t+\int_{G_{T}^{-}} F w d x d t-\int_{S_{T}^{-}} \frac{\partial g}{\partial \aleph} w d s .
\end{gathered}
$$

From (5.5)-(5.7) it follows that

$$
\begin{aligned}
\int_{G_{T}^{-}}\left[-u_{t} w_{t}+\nabla u \nabla w\right] d x d t= & -\int_{G_{T}^{-}} f(u) w d x d t \\
& +\int_{G_{T}^{-}} F w d x d t-\int_{S_{T}^{-}} \frac{\partial g}{\partial \aleph} w d s
\end{aligned}
$$

Finally, summing the equalities (5.4) and (5.8) we receive (5.3).

Let us use the method of test functions [9, pp. 10-12].

In the integral equality (5.3) for test vector-function $w$ we choose $w=$ $\left(l_{1} \psi, \ldots, l_{N} \psi\right)$, where $\psi=\psi_{0}\left[2 T^{-2}\left(t^{2}+|x|^{2}\right)\right]$, while a scalar function $\psi_{0} \in$ $C^{2}(\mathbb{R})$ satisfies the following conditions: $\psi_{0} \geq 0, \psi_{0}^{\prime} \leq 0 ; \psi(\sigma)=1$ for $0 \leq$ $\sigma \leq 1$ and $\psi(\sigma)=0$ for $\sigma \geq 2$ [9, p. 22]. For the chosen test function $w$, using notations $v=\sum_{i=1}^{N} l_{i} u_{i}, g_{0}=\sum_{i=1}^{N} l_{i} g_{i}, F_{*}=\sum_{i=1}^{N} l_{i} F_{i}, f_{0}=\sum_{i=1}^{N} l_{i} f_{i}$, the integral equality (5.3) takes the following form

$$
\begin{aligned}
\int_{G_{T}}\left[-v_{t} \psi_{t}+\nabla v \nabla \psi\right] d x d t= & -\int_{G_{T}} f_{0}(u) \psi d x d t \\
& +\int_{G_{T}} F_{*} \psi d x d t-\int_{S_{T}^{-} \cup S_{T}} \frac{\partial g_{0}}{\partial \aleph} \psi d s .
\end{aligned}
$$


Due to $\left.\psi\right|_{t \geq T}=0$ and the equality $\left.v\right|_{S_{T}^{-} \cup S_{T}}=g_{0}$ in the sense of the trace theory, integrating by parts the left side of the equality (5.9), we get

$$
\begin{aligned}
\int_{G_{T}}\left[-v_{t} \psi_{t}+\nabla v \nabla \psi\right] d x d t & =\int_{G_{T}} v \square \psi d x d t-\int_{S_{T}^{-} \cup S_{T}} v \frac{\partial \psi}{\partial \aleph} d s \\
& =\int_{G_{T}} v \square \psi d x d t-\int_{S_{T}^{-} \cup S_{T}} g_{0} \frac{\partial \psi}{\partial \aleph} d s
\end{aligned}
$$

From (5.9) and (5.10), in view of (5.1) and $\psi \geq 0$, we have

$$
\begin{aligned}
\int_{G_{T}} v \square \psi d x d t \geq & \int_{G_{T}}\left[c_{1}|v|^{\beta}-c_{0}\right] \psi d x d t+\int_{G_{T}} F_{*} \psi d x d t+\int_{S_{T}^{-} \cup S_{T}} g_{0} \frac{\partial \psi}{\partial \aleph} d s \\
& -\int_{S_{T}^{-} \cup S_{T}} \frac{\partial g_{0}}{\partial \aleph} \psi d s=c_{1} \int_{G_{T}}|v|^{\beta} \psi d x d t+\int_{G_{T}}\left(F_{*}-c_{0}\right) \psi d x d t \\
& +\int_{S_{T}^{-} \cup S_{T}} g_{0} \frac{\partial \psi}{\partial \aleph} d s-\int_{S_{T}^{-} \cup S_{T}} \frac{\partial g_{0}}{\partial \aleph} \psi d s .
\end{aligned}
$$

In view of the properties of the function $\psi$ and the inequalities (5.2) we have

$$
\begin{gathered}
\left.\frac{\partial \psi}{\partial \aleph}\right|_{S_{T}^{-} \cup S_{T}} \geq 0, \int_{S_{T}^{-} \cup S_{T}} g_{0} \frac{\partial \psi}{\partial \aleph} d s \geq 0, \\
\int_{S_{T}^{-} \cup S_{T}} \frac{\partial g_{0}}{\partial \aleph} \psi d s \leq 0, \quad \int_{G_{T}} F_{0} \psi d x d t \geq 0,
\end{gathered}
$$

where $F_{0}=F_{*}-c_{0}=\sum_{i=1}^{N} l_{i} F_{i}-c_{0}$. In derivation of the inequality (5.12) we took into account that $\left.\nu_{n+1}\right|_{S_{T}^{-} \cup S_{T}}<0$.

Assuming that the functions $F, g$ and $\psi$ are fixed, introduce into consideration a function of one variable

$$
\gamma(T)=\int_{G_{T}} F_{0} \psi d x d t+\int_{S_{T}^{-} \cup S_{T}} g_{0} \frac{\partial \psi}{\partial \aleph} d s-\int_{S_{T}^{-} \cup S_{T}} \frac{\partial g_{0}}{\partial \aleph} \psi d s, \quad T>0 .
$$

Because of the absolute continuity of integral and the inequalities (5.12), the function $\gamma(T)$ from (5.13) is nonnegative, continuous and nondecreasing function and

$$
\lim _{T \rightarrow 0} \gamma(T)=0
$$

Besides, since according to supposition at least one from the functions $\left.\frac{\partial g_{0}}{\partial \aleph}\right|_{S_{\infty}^{-} \cup S_{\infty}}$ or $F_{0}$ is nontrivial, then

$$
\lim _{T \rightarrow+\infty} \gamma(T)>0
$$

In view of (5.13) the inequality (5.11) can be rewritten as follows

$$
c_{1} \int_{G_{T}}|v|^{\beta} \psi d x d t \leq \int_{G_{T}} v \square \psi d x d t-\gamma(T) .
$$


If in Young's inequality with the parameter $\varepsilon>0$

$$
a b \leq \frac{\varepsilon}{\beta} a^{\beta}+\left(\beta^{\prime} \varepsilon^{\beta^{\prime}-1}\right)^{-1} b^{\beta},
$$

where $\beta^{\prime}=\beta /(\beta-1)$, we take $a=|v| \psi^{1 / \beta}, b=|\square \psi| / \psi^{1 / \beta}$, then taking into account equality $\frac{\beta^{\prime}}{\beta}=\beta^{\prime}-1$, we have

$$
|v \square \psi|=|v| \psi^{1 / \beta} \frac{|\square \psi|}{\psi^{1 / \beta}} \leq \frac{\varepsilon}{\beta}|v|^{\beta} \psi+\frac{1}{\beta^{\prime} \varepsilon^{\beta^{\prime}-1}} \frac{|\square \psi|^{\beta^{\prime}}}{\psi^{\beta^{\prime}-1}} .
$$

In view of (5.17) from (5.16) we have

$$
\left(c_{1}-\frac{\varepsilon}{\beta}\right) \int_{G_{T}}|v|^{\beta} \psi d x d t \leq \frac{1}{\beta^{\prime} \varepsilon^{\beta^{\prime}-1}} \int_{G_{T}} \frac{|\square \psi|^{\beta^{\prime}}}{\psi^{\beta^{\prime}-1}} d x d t-\gamma(T),
$$

whence for $\varepsilon<c_{1} \beta$ we obtain

$$
\int_{G_{T}}|v|^{\beta} \psi d x d t \leq \frac{\beta}{\left(c_{1} \beta-\varepsilon\right) \beta^{\prime} \varepsilon^{\beta^{\prime}-1}} \int_{G_{T}} \frac{|\square \psi|^{\beta^{\prime}}}{\psi^{\beta^{\prime}-1}} d x d t-\frac{\beta}{c_{1} \beta-\varepsilon} \gamma(T) .
$$

Taking into account inequalities $\beta^{\prime}=\beta /(\beta-1), \beta=\beta^{\prime} /\left(\beta^{\prime}-1\right)$ and also equality

$$
\min _{0<\varepsilon<c_{1} \beta} \frac{\beta}{\left(c_{1} \beta-\varepsilon\right) \beta^{\prime} \varepsilon^{\beta^{\prime}-1}}=\frac{1}{c_{1}^{\beta^{\prime}}},
$$

which is reached for $\varepsilon=c_{1}$, then from (5.18) it follows that

$$
\int_{G_{T}}|v|^{\beta} \psi d x d t \leq \frac{1}{c_{1}^{\beta^{\prime}}} \int_{G_{T}} \frac{|\square \psi|^{\beta^{\prime}}}{\psi^{\beta^{\prime}-1}} d x d t-\frac{\beta^{\prime}}{c_{1}} \gamma(T) .
$$

According to the properties of the function $\psi_{0}$, the test function $\psi(x, t)=$ $\psi_{0}\left[2 T^{-2}\left(t^{2}+|x|^{2}\right)\right]=0$ for $r=\left(t^{2}+|x|^{2}\right)^{1 / 2}>T$. Therefore after substitution of variables $t=\frac{1}{\sqrt{2}} T \xi_{0}, x=\frac{1}{\sqrt{2}} T \xi$ we have

$$
\int_{G_{T}} \frac{|\square \psi|^{\beta^{\prime}}}{\psi^{\beta^{\prime}-1}} d x d t=\int_{\substack{r \\ t>|x|}}=\left(t^{2}+|x|^{2}\right)^{1 / 2}<T \frac{|\square \psi|^{\beta^{\prime}}}{\psi^{\beta^{\prime}-1}} d x d t=\left(\frac{1}{\sqrt{2}} T\right)^{n+1-2 \beta^{\prime}} \mathfrak{x}_{0} .
$$

Here

$$
æ_{0}:=\int_{\substack{1<\left|\xi_{0}\right|^{2}+|\xi|^{2}<2 \\ \xi_{0}>|\xi|}} \frac{\left|2(1-n) \psi_{0}^{\prime}+4\left(\xi_{0}^{2}-|\xi|^{2}\right) \psi_{0}^{\prime \prime}\right|^{\beta^{\prime}}}{\psi_{0}^{\beta^{\prime}-1}} d \xi d \xi_{0}<+\infty .
$$

As it is known the test function $\psi(x, t)=\psi_{0}\left[2 T^{-2}\left(t^{2}+|x|^{2}\right)\right]$ with properties mentioned above, for which the condition (5.21) is valid, exists [9, p. 22]. 
Due to (5.20), from the inequality (5.19) and equality $\psi_{0}(\sigma)=1$, for $0 \leq \sigma \leq 1$, we have

$$
\int_{r \leq T / \sqrt{2}}|v|^{\beta} d x d t \leq \int_{D_{T}}|v|^{\beta} \psi d x d t \leq \frac{\left(\frac{1}{\sqrt{2}} T\right)^{n+1-2 \beta^{\prime}}}{c_{1}^{\beta^{\prime}}} \aleph_{0}-\frac{\beta^{\prime}}{c_{1}} \gamma(T) .
$$

When $\beta<(n+1) /(n-1)$, i.e. when $n+1-2 \beta^{\prime}<0$, the equation

$$
\lambda(T)=\frac{\left(\frac{1}{\sqrt{2}} T\right)^{n+1-2 \beta^{\prime}}}{c_{1}^{\beta^{\prime}}} æ_{0}-\frac{\beta^{\prime}}{c_{1}} \gamma(T)=0
$$

has a unique positive root $T=T_{0}(F, g)$, since the function

$$
\lambda_{1}(T)=\left(\left(\frac{1}{\sqrt{2}} T\right)^{n+1-2 \beta^{\prime}} / c_{1}^{\beta^{\prime}}\right) æ_{0}
$$

is a positive, continuous, strictly decreasing function on the interval $(0,+\infty)$, besides, $\lim _{T \rightarrow 0} \lambda_{1}(T)=+\infty$ and $\lim _{T \rightarrow+\infty} \lambda_{1}(T)=0$, and function $\gamma(T)$, as it is stated above, is a nonnegative, continuous and nondecreasing function, satisfying the conditions (5.14) and (5.15). Besides, $\lambda(T)<0$ for $T>T_{0}$ and $\lambda(T)>0$ for $0<T<T_{0}$. Therefore, for $T>T_{0}$ the right side of the inequality (5.22) is negative value, which is impossible. This contradiction proves the Theorem 5.1

Remark 5.1. Let us consider one class of vector-functions $f$, satisfying the condition (5.1):

$$
f_{i}\left(u_{1}, \ldots, u_{N}\right)=\sum_{j=1}^{N} a_{i j}\left|u_{j}\right|^{\beta_{i j}}+b_{i}, \quad i=1, \ldots, N
$$

where $a_{i j}=$ const $>0, b_{i}=$ const, $1<\beta_{i j}=$ const $<\frac{n+1}{n-1} ; i, j=1, \ldots, N$. In this case we can assume that $l_{1}=l_{2}=\ldots=l_{N}=-1$. Indeed, let us choose $\beta=$ const in such a way that $1<\beta<\beta_{i j} ; i, j=1, \ldots, N$. Then it is easy to verify that $|s|^{\beta_{i j}} \geq|s|^{\beta}-1 \forall s \in(-\infty,+\infty)$. Using the inequality [29, p. 302]

$$
\sum_{i=1}^{N}\left|y_{i}\right|^{\beta} \geq N^{1-\beta}\left|\sum_{i=1}^{N} y_{i}\right|^{\beta} \forall y=\left(y_{1}, \ldots, y_{N}\right) \in \mathbb{R}^{N}, \beta=\text { const }>1,
$$

we have

$$
\begin{gathered}
\sum_{i=1}^{N} f_{i}\left(u_{1}, \ldots, u_{N}\right) \geq a_{0} \sum_{i, j=1}^{N}\left|u_{j}\right|^{\beta_{i j}}+\sum_{i=1}^{N} b_{i} \geq a_{0} \sum_{i, j=1}^{N} \mid\left(\left.u_{j}\right|^{\beta}-1\right)+\sum_{i=1}^{N} b_{i} \\
=a_{0} N \sum_{j=1}^{N}\left|u_{j}\right|^{\beta}-a_{0} N^{2}+\sum_{i=1}^{N} b_{i} \geq a_{0} N^{2-\beta}\left|\sum_{j=1}^{N} u_{j}\right|^{\beta}+\sum_{i=1}^{N} b_{i}-a_{0} N^{2}, \\
a_{0}=\min _{i, j} a_{i j}>0 .
\end{gathered}
$$


Whence it follows the inequality (5.1), where

$$
l_{1}=l_{2}=\ldots=l_{N}=-1, c_{0}=a_{0} N^{2}-\sum_{i=1}^{N} b_{i}, c_{1}=a_{0} N^{2-\beta}>0 .
$$

Note that the vector-function $f$, represented by the equalities (5.24), also satisfies the condition (5.1) for $l_{1}=l_{2}=\ldots=l_{N}=-1$ for less restrictive conditions, when $a_{i j}=$ const $\geq 0$, but $a_{i k_{i}}>0$, where $k_{1}, \ldots, k_{N}$ is any arbitrary fixed permutation of the numbers $1,2, \ldots, N ; i, j=1, \ldots, N$.

Remark 5.2. From the Theorem 5.1 it follows that when its conditions are fulfilled, then the problem (1.1), (1.2) can not have a global strong generalized solution of the class $W_{2}^{1}$ in the domain $D_{\infty}$ in the sense of the Definition 2.4.

\section{References}

[1] Bitsadze, A.V.: Some Classes of Partial Differential Equations. Nauka (Russian), Moscow (1981)

[2] Jörgens, K.: Das Anfangswertproblem im Grossen fur eine Klasse nichtlinearer Wellengleichungen. Math. Z. 77, 295-308 (1961)

[3] Segal, L.E.: The global Cauchy problem for a relativistic scalar field with power interaction. Bull. Soc. Math. France 91, 129-135 (1963)

[4] Levine, H.A.: Instability and nonexistence of global solutions to nonlinear wave equations of the form $P u_{t t}=-A u+F(u)$. Trans. Am. Math. Soc. 192, 1-21 (1974)

[5] John, F.: Blow-up of solutions of nonlinear wave equations in three space dimensions. Manuscripta Math. 28(1-3), 235-268 (1979)

[6] Kato, T.: Blow-up of solutions of some nonlinear hyperbolic equations. Commun. Pure Appl. Math. 33(4), 501-505 (1980)

[7] Sideris, T.C.: Nonexistence of global solutions to semilinear wave equations in high dimensions. J. Differ. Equ. 52(3), 378-406 (1984)

[8] Georgiev, V., Lindblad, H., Sogge, C.D.: Weighted Strichartz estimates and global existence for semilinear wave equations. Am. J. Math. 119(6), 12911319 (1997)

[9] Mitidieri, È., Pohozhaev, S.I.: A priori estimates and the absence of solutions of nonlinear partial differential equations and inequalities. (Russian) Trudy Mat. Inst. Steklova, 234 (2001), 1-384; English transl.: Proc. Steklov Inst. Math. 234(3), 1-362 (2001)

[10] Ikehata, R., Tanizawa, K.: Global existence of solutions for semilinear damped wave equations in $R^{n}$ with noncompactly supported initial data. Nonlinear Anal. 61, 1189-1208 (2005) 
[11] Zhou, Y.: Global existence and nonexistence for a nonlinear wave equation with damping and source terms. Math. Nachr. 278(11), 1341-1358 (2008)

[12] Bitsadze, A.V.: Mixed type equations on three-dimensional domains. (Russian) Dokl. Akad. Nauk SSSR 143(5), 1017-1019 (1962)

[13] Vragov, V.N.: The Goursat and Darboux problems for a certain class of hyperbolic equations.. (Russian) Differential'Nye Uravneniya 8(1), 7-16 (1972)

[14] Vragov, V.N.: Boundary value problems for nonclassical equations of mathematical physics. Novosibirsk State University (Russian), Novosibirsk (1983)

[15] Nakhushev, A.M.: A multidimensional analogue of the Darboux problem for hyperbolic equations. (Russian) Dokl. Akad. Nauk SSSR 194, 31-34 (1970)

[16] Nakhushev, A.M.: Equations of mathematical biology. Vysshaya Shkola (Russian), Moscow (1995)

[17] Kalmenov, T.Sh.: On multidimensional regular boundary value problems for the wave equation. (Russian) Izv. Akad. Nauk Kazakh. SSR. Ser. Fiz.-Mat., no. 3, $18-25(1982)$

[18] Kharibegashvili, S.: On the solvability of one multidimensional version of the first Darboux problem for some nonlinear wave equations. Nonlinear Anal. 68(4), 912-924 (2008)

[19] Bogveradze, G., Kharibegashvili, S.: On the global and local solution of the multidimensional Darboux problem for some nonlinear wave equations. Georgian Math. J. 14(1), 65-80 (2007)

[20] Kharibegashvili, S., Midodashvili, B.: On the solvability of one boundary value problem for some semilinear wave equations with source terms. Nonlinear Differ. Equ. Appl. 18, 117-138 (2011)

[21] Ladyzhenskaya, O.A.: Boundary Value Problems of Mathematical Physics. Nauka (Russian), Moscow (1973)

[22] Krasnosel'skiǐ, M.A., Zabreǐko, P.P., Pustyl'nik, E.I., Sobolevskǐ̌, P.E.: Integral Operators in Spaces of Summable Functions. Nauka (Russian), Moscow (1966)

[23] Kufner, A., Futchik, S.: Nonlinear differential equations. (Translated into Russian) Nauka, Moscow (1988); English original: Studies in Applied Mechanics, vol. 2. Elsevier Scientific Publishing Co., Amsterdam (1980)

[24] Kharibegashvili, S.: Some multidimensional problems for hyperbolic partial differential equations and systems. Mem. Differ. Equ. Math. Phys. 37, 1-136 (2006)

[25] Henry, D.: Geometrical theory of semi-linear parabolic equations. (Translated into Russian) Mir, Moscow (1985); English original: Lecture Notes in Mathematics, vol. 840. Springer, Berlin (1981)

[26] Hörmander, L.: Linear partial differential operators. (Translated into Russian) Mir, Moscow (1965); English original: die Grundlehren der mathematischen 
Vol. 20 (2013) The Darboux problem version for hyperbolic systems

Wissenschaften, Bd. 116. Academic Press Inc., Publishers, New York; Springer, Berlin (1963)

[27] Trenogin, V.A.: Functional Analysis. Nauka (Russian), Moscow (1993)

[28] Vulikh, B.Z.: Concise course of the theory of functions of a real variable. Nauka (Russian), Moscow (1973)

[29] Fichtengolz, G.M.: Course of differential and integral calculus. I. Nauka (Russian), Moscow (1969)

S. Kharibegashvili

A. Razmadze Mathematical Institute

I. Javakhishvili Tbilisi State University

2, University St.

0143 Tbilisi

Georgia

e-mail: kharibegashvili@yahoo.com

S. Kharibegashvili

Department of Mathematics

Georgian Technical University

77, M. Kostava Str.

0175 Tbilisi

Georgia

S. Midodashvili

Faculty of Exact and Natural Sciences

I. Javakhishvili Tbilisi State University

2, University St.

0143 Tbilisi

Georgia

B. Midodashvili

Faculty of Education, Exact and Natural Sciences

Gori Teaching University

53, Chavchavadze Str.

Gori

Georgia

e-mail: bidmid@hotmail.com

Received: 9 October 2011.

Accepted: 25 April 2012. 